\title{
Optimal Design of Autonomous Vehicle Zones in Transportation NETWORKS
}

\author{
Zhibin Chen ${ }^{\mathrm{a}}$, Fang $\mathrm{He}^{\mathrm{b}}$, Yafeng $\mathrm{Yin}^{\mathrm{c}}$, a* and Yuchuan $\mathrm{Du}^{\mathrm{c}}$ \\ ${ }^{a}$ Department of Civil and Coastal Engineering, University of Florida, 365 Weil Hall, \\ Gainesville, FL 32611-6580, United States \\ ${ }^{b}$ Department of Industrial Engineering, Tsinghua University, N502 Shunde Building, \\ Beijing, 100084, P.R. China \\ 'School of Transportation Engineering, Tongji University, Shanghai, China, 201804
}

\begin{abstract}
This paper advocates the need for infrastructure planning to adapt to and further promote the deployment of autonomous vehicle (AV) technology. It is envisioned that in the future government agencies will dedicate certain areas of road networks to AVs only to facilitate the formulation of vehicle platoons to improve throughput and hopefully improve the performance of the whole network. This paper aims to present a mathematical framework for the optimal design of AV zones in a general network. With the presence of AV zones, AVs may apply different routing principles outside of and within the AV zones. A novel network equilibrium model (we refer to it as the "mixed routing equilibrium model") is thus firstly proposed to capture such mixed-routing behaviors. We then proceed to formulate a mixed-integer bi-level programming model to optimize the deployment plan of AV zones. Numerical examples are presented to demonstrate the performance of the proposed models.
\end{abstract}

Keywords: autonomous vehicle, autonomous-vehicle zone, mixed routing equilibrium, optimal deployment

*Corresponding author. Tel.: 352-392-9537 Ext. 1455; Email: yafeng@ufl.edu 


\section{INTRODUCTION}

Autonomous vehicles (AVs) are expected to offer extraordinary improvements to both the safety and efficiency of existing roadways and mobility systems. Although it will be many years before a widespread adoption of AV technology, recent developments suggest that they are fastapproaching. Google's AVs had driven more than 2,000,000 miles on public roads by October 2016 (Google Self-Driving Car Project, 2016). More recently, nuTonomy, a software company, has launched the world's first self-driving taxi in Singapore (nuTonomy, 2016). Many car manufactures, such as Volvo and Audi, are currently designing and testing their prototype AVs. In the United States, states such as Nevada, Florida, California, Michigan, and Washington D.C. have legalized AVs for testing on public roads. While thus far the development of AV technology appears to be primarily driven by the private sector, it is critical for government agencies to change various policies and practices to adapt to and further promote the deployment of the technology.

In this paper, we advocate the need for infrastructure adaptation planning for AVs. Before manual driving can be completely phased out (or criminalized, as some have predicted), the traffic stream on a road network will still be heterogeneous, with both conventional vehicles (CVs) and AVs. We envision that government agencies can initially identify critical locations to implement various AV mobility applications. For example, a "bottleneck manager" can be implemented at a recurrent freeway bottleneck. When approaching, AVs send requests via vehicle-to-infrastructure wireless commutations to the "bottleneck manager," which will prioritize the requests and optimize their trajectories to ensure timely passage while preventing the bottleneck from being activated. To leverage the growing adoption of AVs, government agencies may later dedicate certain traffic lanes, highway segments or even areas of networks exclusively to AVs to facilitate the formulation of vehicle platoons to further improve throughput. Subsequently implemented are innovative control strategies that aim to achieve system optimum in those areas. The dedicated AV areas will expand gradually as the level of the market penetration of AVs increases and eventually support a fully connected and automated mobility in the whole system. Similar ideas have been suggested in the literature. For example, as current managed lanes are equipped with advanced communication and data transfer systems, researchers have suggested converting some of them into dedicated lanes for AVs to reduce congestion and improve the safety of passengers (Davis, 2014; Levin and Boyles, 2016a,b). To help boost the market penetration of AVs, Chen et al. (2016) proposed a time-dependent model to optimally deploy AV lanes on a general network consisting of both CVs and AVs. Godsmark and Kakkar (2014) pointed out that the presence of AV areas can maximize benefits brought by AVs as rapidly as possible, as well as promote the AV adoption.

This paper deals with a particular issue in the above infrastructure adaptation planning process and aims to present a mathematical framework for the optimal design of AV zones in a general network. With only AVs being allowed to enter, an AV zone consists of a set of links that are tailored to AVs. Note that in order not to compromise CVs' accessibility to various locations, the nodes within the zone in particular, the AV zone can be designed to consist of only urban expressways or arterial roads, excluding minor streets. It is assumed that within the zone, AVs cannot choose their routes. Instead, they report their exits and are then guided by a central controller to achieve the system optimum flow distribution in the zone. AV zones will enable full 
utilization of the AV technology within the zones to hopefully improve the performance of the whole network. These zones can help reduce travel times for AVs and further nurture the AV market. However, the existence of AV zones likely increases travel times for some CVs. Therefore, government agencies will need to make a tradeoff between these pros and cons in designing AV zones. The optimal design will depend on the market penetration of AVs, network topology and link characteristics, and more importantly, the route choices of both CVs and AVs in the network.

Optimal design of AV zones possesses a structure of the leader-follower or Stackelberg game, in which government agencies serve as the leader while CVs and AVs are the followers. Given a design of the AV zone, this paper firstly develops an innovative user equilibrium model we call the "mixed routing equilibrium model" to describe the flow distribution of AVs and CVs across the network. The novelty of the proposed model lies in the aspect that some paths consist of both links outside of and within the AV zones; AVs follow the user-optimum routing principle in the former and a different routing principle such as the system-optimum one in the latter. This new equilibrium model is most relevant to mixed equilibrium models in the literature, e.g., Haurie and Marcotte (1985), Harker (1988), Yang and Zhang (2008), Zhang et al. (2008), and He et al. (2013), where both the user-optimum and system-optimum route choice behaviors are considered. In all these previous models, all types of players share the same network, and each type of player applies a particular routing principle to traverse the whole network. In contrast, in our model, AVs and CVs may face different network topologies (recall that CVs are not allowed to enter AV zones) and, more importantly, AVs may apply different routing principles at different sub-networks. Mixed routing behaviors may become more relevant with the deployment of automated and connected vehicles. Capturing them in the network equilibrium framework is very challenging, which actually constitutes one of the major contributions of this paper.

Given the proposed mixed routing equilibrium model, we proceed to optimize the deployment plan of AV zones over a general network. The design problem is formulated as a mixed-integer bi-level programing model that is very difficult to solve. The problem appears to have a similar structure as the cordon design problem for cordon congestion pricing (see, e.g., Zhang and Yang, 2004 and Sumalee, 2004), which was solved previously using genetic-algorithm-based heuristics, such as the cutset-based approach (Zhang and Yang, 2004), the branch-tree approach (Sumalee, 2004), and the Delaunay triangulation approach (Hult, 2006). However, most of the above algorithms have low efficiency on generating new feasible design plans. In this study, we adopt a simulated annealing algorithm or SAA (Kirkpatrick et al., 1983; Cerny, 1985) to solve the AV zone design problem, since a simple but efficient plan-updating strategy can be tailored for SAA in order to generate new feasible design plans efficiently.

For the remainder, Section 2 illustrates the operational concept of AV zones considered in this paper and basic assumptions for the proposed models. Section 3 formulates the mixed routing equilibrium model and proposes its solution algorithm. Further, Section 4 optimizes the design of AV zones, and Section 5 concludes the paper. 


\section{PROBLEM DESCRIPTION}

We consider a network where both AVs and CVs are present. The origin-destination (O-D) matrices of the vehicular trips of AVs and CVs are considered given. It is envisioned that a government agency strategically designs AV zones on a road network. An AV zone is cordoned off by a virtual loop. See FIGURE 1 for an example of where the nodes and links within loop C comprise an AV zone. To facilitate the presentation of the model formulation, this study hereinafter considers the deployment or presence of a single AV zone over the network, but the proposed model can be easily extended to the case with multiple AV zones directly. Below we illustrate the operational concept for the AV zone:

i. Only AVs are allowed to use the links within the zone;

ii. When entering the zone, AVs must report their exits of the zone to the control center, which routes AVs to traverse the zone;

iii. Based on AVs' entrances and exits, the control center routes AVs to minimize the total travel time in the zone ${ }^{1}$.

In the presence of an $\mathrm{AV}$ zone, when making their route choices, CVs need to avoid the zone while AVs will decide whether to access the zone, and where to enter and exit. Note that among all paths connecting the same O-D pair, some may traverse the AV zone while others will not. When comparing these paths, AVs need to perceive the times spent within and outside of the AV zone.

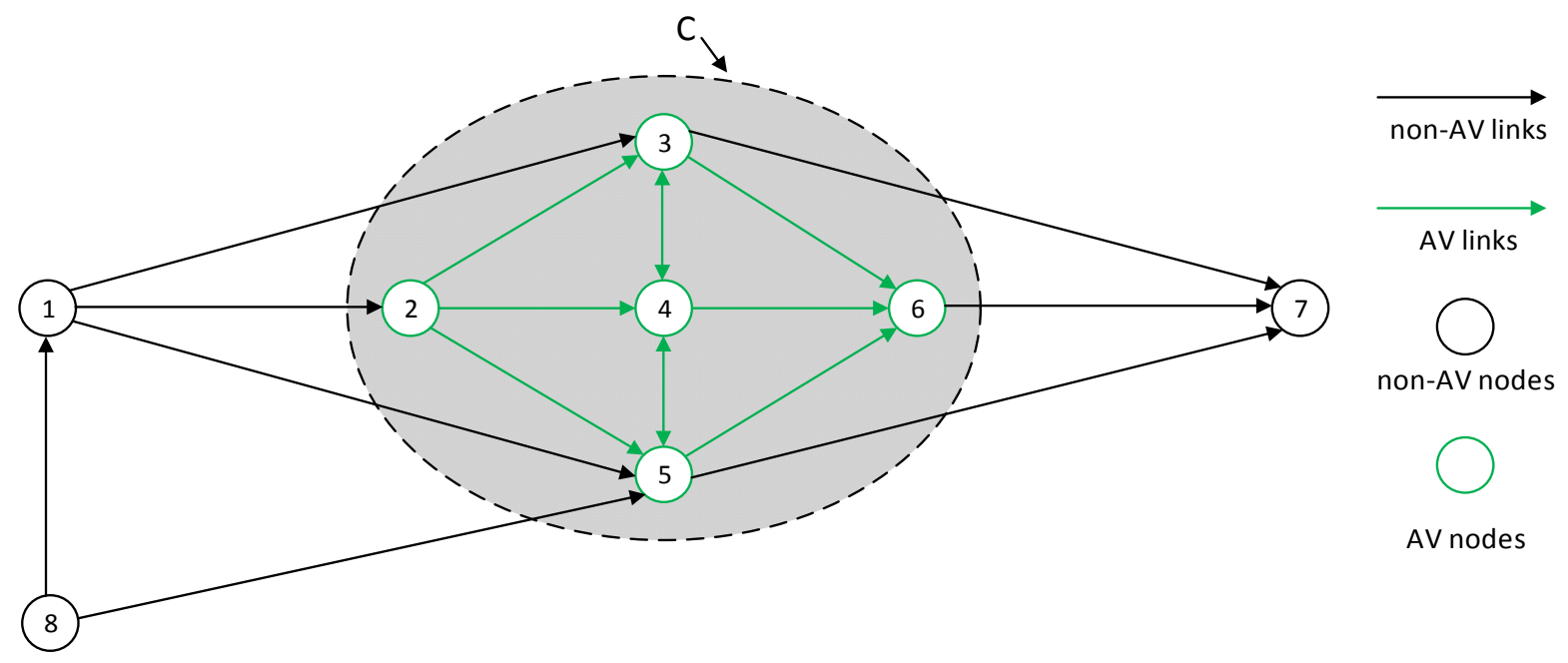

FIGURE 1 An example of AV zone

Since the overarching goal of this study is AV zone planning, a static deterministic modeling framework is adopted. Below we summarize basic assumptions for our model formulations:

i. AVs using the same entrance and exit of the AV zone may experience different travel times due to system-optimum routing. We assume that AVs perceive their travel times to be the minimum travel times between their corresponding entrances and exits of the AV zone.

\footnotetext{
${ }^{1}$ See Section 3.7 for a discussion of other routing principles.
} 
ii. All vehicles are assumed to minimize their own perceived trip times.

iii. The per-lane capacity of links within the AV zone is much larger than those of regular links due to vehicle automation (see, e.g., Kesting et al, 2010; Shladover et al., 2012; Levin and Boyles, 2015).

iv. The capacity of a regular link with mixed traffic of CVs and AVs remains the same as when only CVs use the link.

v. The performance functions of regular and AV links may be different, but all are increasing functions with link flows.

vi. In the network equilibrium model, there exists at least one usable path between each O-D pair for both AVs and CVs. When designing the AV zone, if certain inner nodes within the zone are origins or destinations, the corresponding demands of CVs will be discarded for equilibrium analysis, because no feasible path will exist for CVs between these O-D pairs. Subsequently, the resulting loss of social welfare will be calculated as part of the social cost, which is the objective that government agencies aim at minimizing.

It is worth noting here that the equilibrium model developed in this paper is different from previous network equilibrium analyses of AV flows, e.g., Correia and van Arem (2016), which focus on shared-use automated mobility and explicitly consider automated vehicle routing to meet the travel demands of households; all households act selfishly in choosing their paths and schedules. In contrast, this paper assumes that the vehicular O-D pattern of AVs is known and focuses on modeling the mixed routing behaviors that arise with the existence of AV zones.

For the convenience of readers, below we list notations frequently used in the paper.

\begin{tabular}{ll}
\hline Sets & \\
\hline$N$ & Set of regular nodes \\
$A$ & Set of regular links \\
$\widetilde{N}$ & Set of AV nodes \\
$\tilde{A}$ & Set of AV links \\
$\widetilde{N}$ & Set of dummy AV nodes \\
$\hat{A}$ & Set of dummy AV links \\
$W$ & Set of origin-destination (O-D) pairs \\
$\widetilde{W}$ & Set of entrance-exit (E-E) pairs for the AV zone/network \\
$M$ & Set of modes or classes, including CVs and AVs \\
$P^{w, m}$ & Set of paths between O-D pair $w \in W$ by mode $m \in M$ \\
$A(p)$ & Set of links along path $p \in P^{w, m}$ between O-D pair $w \in W$ by mode $m \in M$ \\
\hline Parameters & \\
\hline$a$ & Link $a=(i, j) \in A \cup \hat{A}$ on the revised network \\
$\tilde{a}$ & Link $\tilde{a}=(i, j) \in \tilde{A}$ on the AV network \\
$w$ & O-D pair $w \in W$ \\
$\widetilde{w}$ & E-E pair $\widetilde{w} \in \widetilde{W}$ \\
\hline
\end{tabular}




\begin{tabular}{ll}
\hline$m$ & Mode $m \in M$ \\
$d^{w, m}$ & Travel demand between O-D pair $w \in W$ by mode $m \in M$ \\
$o(\widetilde{w})$ & Entrance of E-E pair $\widetilde{w} \in \widetilde{W}$ \\
$d(\widetilde{w})$ & Exit of E-E pair $\widetilde{w} \in \widetilde{W}$ \\
$p$ & Path $p \in P^{w, m}$ between O-D pair $w \in W$ by mode $m \in M$ \\
\hline Variables & Traffic flow of link $a \in A \cup \hat{A}$ \\
\hline$v_{a}$ & Traffic flow of link $\tilde{a} \in \tilde{A}$ \\
$v_{\tilde{a}}$ & Link flow on link $a \in A \cup \hat{A}$ for O-D pair $w \in W$ by mode $m \in M$ \\
$x_{a}^{w, m}$ & Link flow on link $\tilde{a} \in \tilde{A}$ for E-E pair $\widetilde{w} \in \widetilde{W}$ \\
$x_{\tilde{a}}^{\widetilde{w}}$ & Travel time of link $a \in A$ specified by the link performance function \\
$t_{a}\left(v_{a}\right)$ & Travel time of link $\tilde{a} \in \tilde{A}$ specified by the link performance function \\
$t_{\tilde{a}}\left(v_{\tilde{a}}\right)$ & Travel time of dummy AV link $a \in \hat{A}$ \\
$c_{a}$ &
\end{tabular}

\section{MIXED ROUTING EQUILIBRIUM MODEL}

Let $\widetilde{G}(\widetilde{N}, \tilde{A})$ denote the network within the AV zone, where $\widetilde{N}$ and $\tilde{A}$ are the sets of nodes and links in the zone, respectively. For convenience, we hereinafter refer to them as AV network, AV nodes, and AV links. Based on $\tilde{G}(\widetilde{N}, \tilde{A})$, we construct a dummy AV network to replace the original AV network. Specifically, such a dummy AV network only consists of those AV nodes that are either entrances or exits of the AV zone (e.g., nodes 2, 3, 5, and 6 in FIGURE 1, and we hereinafter refer to them as dummy AV nodes). Moreover, if an AV node is either an origin or a destination, it will also be regarded as an entrance or an exit of the AV zone. Further, dummy AV links are constructed to specify the connectivity between those nodes. For example, for the AV zone in FIGURE 1, if node 4 is neither an origin nor a destination, then the dummy AV links can be constructed as FIGURE 2(a); otherwise, FIGURE 2(b). By doing so, each dummy AV link represents a set of paths connecting an entrance and an exit. For example, dummy AV link 2-3 in FIGURE 2(a) represents paths $2 \rightarrow 3,2 \rightarrow 4 \rightarrow 3$, and $2 \rightarrow 5 \rightarrow 4 \rightarrow 3$ in FIGURE 1. Consequently, the flow on dummy link 2-3 represents the demand between entrance 2 and exit 3. In addition, as per Assumption i in Section 2, its travel time is equal to the shortest time of paths $2 \rightarrow 3,2 \rightarrow 4 \rightarrow 3$, and $2 \rightarrow 5 \rightarrow 4 \rightarrow 3$ in FIGURE 1. In other words, a dummy AV link can be viewed as the shortest path connecting the associated entrance and exit. Let $\hat{G}(\widehat{N}, \hat{A})$ denote the dummy AV network where $\widehat{N}$ and $\hat{A}$ are the sets of dummy AV nodes and links.

Let $N$ and $A$ denote the sets of non-AV or regular nodes and non-AV or regular links that are nodes and links outside of the AV zone (e.g., nodes 1, 7, and links 1-3, 8-1 in FIGURE 1). Let $G(N \cup \widehat{N}, A \cup \hat{A})$ denote the network containing non-AV nodes, non-AV links, and dummy AV nodes and links (we refer to $G$ as a revised network). For example, if node 4 is neither an origin nor a destination, then FIGURE 3 illustrates the revised network. In a revised network, we only 
need to consider the user-optimum routing principle without worrying about the mixed routing behaviors that exist in the original network.

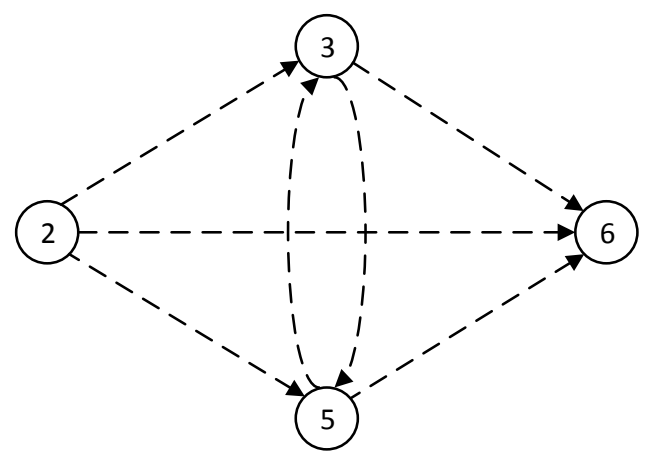

(a)

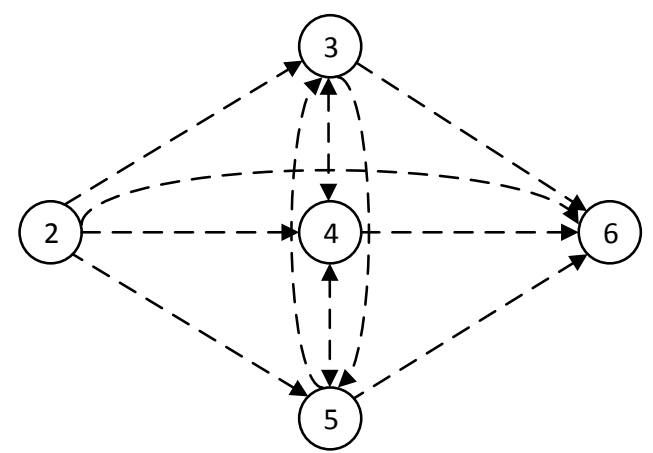

(b)

FIGURE 2 Dummy AV networks

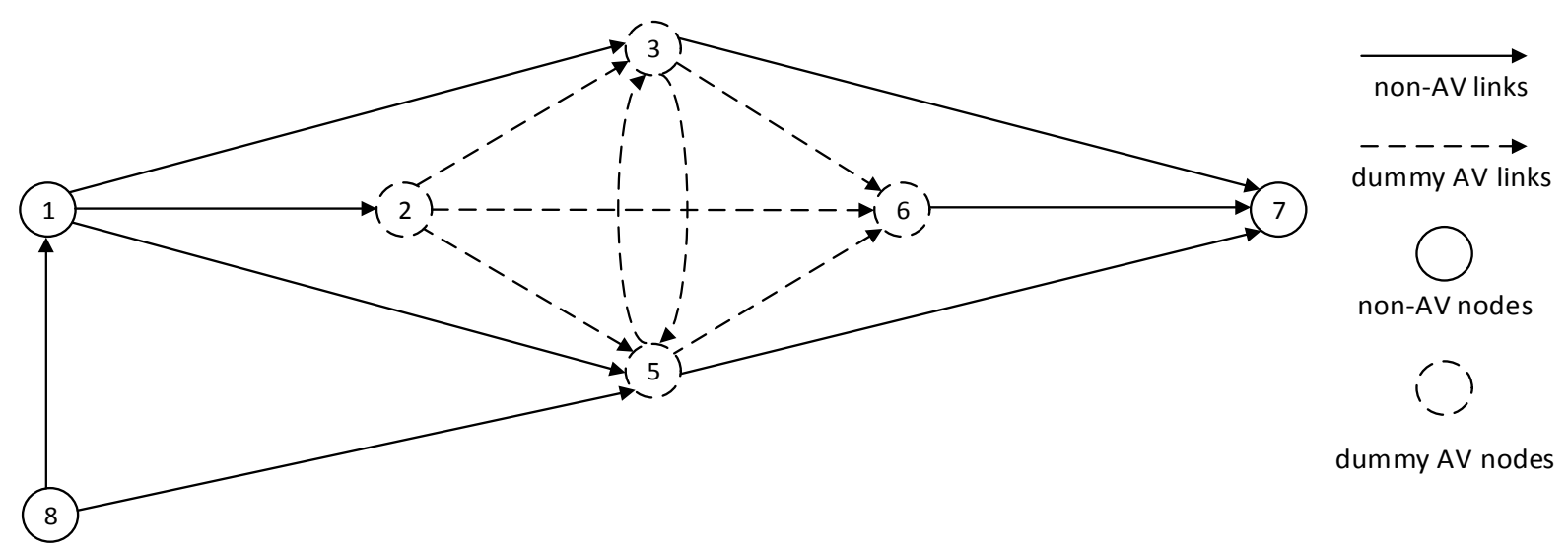

FIGURE 3 A revised network

We represent a link in the revised network as $a \in A \cup \hat{A}$, or its starting and ending nodes, i.e., $a=(i, j) \in A \cup \hat{A}$. Similarly, $\tilde{a} \in \tilde{A}$ represents an AV link. Let $W$ and $\widetilde{W}$ denote the sets of O-D pairs for the revised network (note that these O-D pairs are the same as those in the original network), and entrance-exit (E-E) pairs associated with the AV network. Further, we use $o(\widetilde{w})$ and $d(\widetilde{w})$ to denote the entrance and exit of E-E pair $\widetilde{W} \in \widetilde{W}$. Let $M$ be the set of transportation modes CVs and AVs, i.e., $M=\{C, A\}$. Let $d^{w, m}$ and $P^{w, m}$ represent the travel demand and the set of paths between O-D pair $w \in W$ by mode $m \in M$, respectively. Let $x_{a}^{w, m}$ be the flow on link $a \in$ $A \cup \hat{A}$ for O-D pair $w \in W$ by mode $m \in M$, and $x_{\tilde{a}}^{\widetilde{w}}$ be the flow on link $\tilde{a} \in \tilde{A}$ for E-E pair $\widetilde{w} \in$ $\widetilde{W}$. Let $v_{a}$ and $v_{\tilde{a}}$ be the aggregate flows of links $a \in A \cup \hat{A}$ and $\tilde{a} \in \tilde{A}$. Let $t_{a}\left(v_{a}\right)$ and $t_{\tilde{a}}\left(v_{\tilde{a}}\right)$ define the travel times of links $a \in A$ and $\tilde{a} \in \tilde{A}$ specified by the performance functions of the links. Note that, according to Assumption iii, the per-lane capacity of each link within the AV zone, i.e., $\tilde{a} \in \tilde{A}$, will be substantially improved. Let $c_{a}$ represent the travel time of dummy AV link $a \in$ $\hat{A}$. 


\subsection{Travel time of dummy links}

As previously mentioned, $c_{a}$ is assumed to be equal to the minimum trip time of the corresponding E-E pair $\widetilde{w} \in \widetilde{W}$. Specifically, with a given traffic flow distribution of the AV network, $v_{\tilde{a}}, \forall \tilde{a} \in$ $\tilde{A}$, finding the shortest path can be formulated as follows for each E-E pair $\widetilde{W} \in \widetilde{W}$ :

SP:

$$
\min _{z} \sum_{\tilde{a} \in \tilde{A}} t_{\tilde{a}}\left(v_{\tilde{a}}\right) z_{\tilde{a}}^{\widetilde{w}}
$$

$$
\begin{array}{ll}
\text { s.t. } \tilde{\Delta} \mathbf{z}^{\widetilde{w}}=\tilde{E}^{\widetilde{w}} & \\
z_{\tilde{a}}^{\widetilde{W}} \geq 0 & \forall \tilde{a} \in \tilde{A}
\end{array}
$$

where $\tilde{\Delta}$ is the node-link incidence matrix associated with the AV network and $\tilde{E}^{\widetilde{w}}$ is a vector with a length of $|\widetilde{N}|$. The vector consists of two-nonzero components: one has a value of 1 in the component corresponding to the entrance of $\widetilde{w} \in \widetilde{W}$, and the other has a value of -1 in the component corresponding to the exit of $\widetilde{W} \in \widetilde{W}$.

In the above, the objective function is to minimize the total trip time. Constraint (1) ensures flow balance, and constraint (2) makes sure that $\boldsymbol{Z}$ are nonnegative variables. Since the matrix associated with constraint (1) is totally unimodular, the optimal solution of SP must be integers. Further, based on constraint (1) and the objective function, it is easy to verify that the optimal value of $\boldsymbol{z}$ cannot be greater than 1 . Therefore, the optimal solution $z_{\tilde{a}}^{\widetilde{W} *}, \forall \tilde{a} \in \tilde{A}$, is equal to either 0 or 1 . Specifically, $z_{\tilde{a}}^{\widetilde{\omega} *}=1$ if link $\tilde{a}$ is utilized, and 0 otherwise. Therefore, we can obtain the travel time of dummy AV link $a \in \hat{A}$ as below:

$$
c_{a}=\sum_{\widetilde{w} \in \widetilde{W}} \beta_{a}^{\widetilde{w}}\left[\sum_{\tilde{a} \in \tilde{A}} t_{\tilde{a}}\left(v_{\tilde{a}}\right) z_{\tilde{a}}^{\widetilde{\tilde{a}}^{*}}\right]
$$

where $\beta_{a}^{\widetilde{w}}$ is a binary parameter that equals 1 if the dummy link $a$ corresponds to the E-E pair $\widetilde{w} \in$ $\widetilde{W}$, and 0 otherwise.

SP is a linear program written for each E-E pair $\widetilde{w} \in \widetilde{W}$. Its optimality conditions are stated as follows:

(1)-(2)

$$
\begin{array}{ll}
{\left[t_{\tilde{a}}\left(v_{\tilde{a}}\right)-\kappa_{i}^{\widetilde{w}}+\kappa_{j}^{\widetilde{w}}\right] z_{\tilde{a}}^{\widetilde{w}}=0} & \forall \tilde{a}=(i, j) \in \tilde{A}, \widetilde{w} \in \widetilde{W} \\
t_{\tilde{a}}\left(v_{\tilde{a}}\right)-\kappa_{i}^{\widetilde{w}}+\kappa_{j}^{\widetilde{w}} \geq 0 & \forall \tilde{a}=(i, j) \in \tilde{A}, \widetilde{w} \in \widetilde{W}
\end{array}
$$

where $\boldsymbol{\kappa}$ are the multipliers associated with constraints (1). 


\subsection{User equilibrium flow distribution in the revised network}

As previously mentioned, in the revised network, we only need to consider the user-optimum routing principle. With $c_{a}$ calculated as above for $a \in \hat{A}$, the user equilibrium conditions can be mathematically defined as follows:

$$
\begin{array}{ll}
\Delta \boldsymbol{x}^{w, m}=\boldsymbol{E}^{w, m} d^{w, m} & \forall m \in M, w \in W \\
x_{a}^{w, A} \geq 0 & \forall a \in A \cup \hat{A}, w \in W \\
x_{a}^{w, C} \geq 0 & \forall a \in A, w \in W \\
x_{a}^{w, C}=0 & \forall a \in \hat{A}, w \in W \\
v_{a}=\sum_{w \in W} \sum_{m \in M} x_{a}^{w, m} & \forall a \in A \cup \hat{A} \\
t_{a}\left(v_{a}\right)-\rho_{i}^{w, m}+\rho_{j}^{w, m} \geq 0 & \forall a=(i, j) \in A, w \in W, m \in M \\
c_{a}\left(v_{a}\right)-\rho_{i}^{w, A}+\rho_{j}^{w, A} \geq 0 & \forall a=(i, j) \in \hat{A}, w \in W \\
\left(t_{a}\left(v_{a}\right)-\rho_{i}^{w, m}+\rho_{j}^{w, m}\right) \cdot x_{a}^{w, m}=0 & \forall a=(i, j) \in A, w \in W, m \in M \\
\left(c_{a}\left(v_{a}\right)-\rho_{i}^{w, A}+\rho_{j}^{w, A}\right) \cdot x_{a}^{w, A}=0 & \forall a=(i, j) \in \hat{A}, w \in W
\end{array}
$$

where $\Delta$ is the node-link incidence matrix associated with the revised network and $E^{w}$ is a vector with a length of $|N|$. The vector consists of two-nonzero components: one has a value of 1 in the component corresponding to the origin of $w \in W$, and the other has a value of -1 in the component corresponding to the destination of $w \in W . \boldsymbol{\rho}$ are auxiliary variables representing the node potentials.

In the above, constraint (6) ensures flow balance. Constraints (7) and (8) imply that the link flow between each O-D pair by each mode should be nonnegative, and constraint (9) ensures that only AVs can use dummy AV links. Constraint (10) implies that the aggregate link flow is the summation of link flow between different O-D pairs by different modes. Constraints (11)-(14) are complementary slackness conditions, ensuring that the perceived travel times of utilized paths between an O-D pair for the same mode are the same, but less than or equal to that of any unutilized usable path. Specifically, a path is usable for a mode if all the links along the path are usable for the mode. For example, a path containing any AV link is not usable for CVs.

\subsection{System-optimum routing within the AV network}

As mentioned before, the demand for each E-E pair equals the flow of the corresponding dummy AV link. Specifically, given the flow distribution of the revised network, the flow of a dummy AV link, say $\ddot{a} \in \hat{A}$, is calculated to be $\sum_{w \in W} x_{\ddot{a}}^{w, A}$. Therefore, the system-optimum flow distribution within the $\mathrm{AV}$ zone can be formulated as follows: 


$$
\begin{array}{ll}
\sum_{j} x_{o(\widetilde{w}), j}^{\widetilde{w}}-\sum_{k} x_{k, o(\widetilde{w})}^{\widetilde{w}}=\sum_{a \in \hat{A}} \beta_{a}^{\widetilde{w}} \sum_{w \in W} x_{a}^{w, A} & \forall \widetilde{w} \in \widetilde{W} \\
\sum_{j} x_{d(\widetilde{w}), j}^{\widetilde{w}}-\sum_{k} x_{k, d(\widetilde{w})}^{\widetilde{w}}=-\sum_{a \in \tilde{A}} \beta_{a}^{\widetilde{w}} \sum_{w \in W} x_{a}^{w, A} & \forall \widetilde{w} \in \widetilde{W} \\
\sum_{j}^{\widetilde{w}} x_{i, j}^{\widetilde{w}}-\sum_{k} x_{k, i}^{\widetilde{w}}=0 & \forall i \in \widetilde{N} \backslash\{o(\widetilde{w}), d(\widetilde{w} \\
x_{\tilde{a}}^{\widetilde{w}} \geq 0 & \forall \tilde{a} \in \tilde{A}, \widetilde{w} \in \widetilde{W} \\
v_{\tilde{a}}=\sum_{\widetilde{w} \in \widetilde{W}} x_{\tilde{a}}^{\widetilde{w}} & \forall \tilde{a} \in \tilde{A} \\
t_{\tilde{a}}\left(v_{\tilde{a}}\right)+v_{\tilde{a}} t_{\tilde{a}}^{\prime}\left(v_{\tilde{a}}\right)-\tilde{\rho}_{i}^{\widetilde{w}}+\tilde{\rho}_{j}^{\widetilde{w}} \geq 0 & \forall \tilde{a} \in \tilde{A}, \widetilde{w} \in \widetilde{W} \\
\left(t_{\tilde{a}}\left(v_{\tilde{a}}\right)+v_{\tilde{a}} t_{\tilde{a}}^{\prime}\left(v_{\tilde{a}}\right)-\tilde{\rho}_{i}^{\widetilde{w}}+\tilde{\rho}_{j}^{\widetilde{w}}\right) \cdot x_{\tilde{a}}^{\widetilde{w}}=0 & \forall \tilde{a} \in \tilde{A}, \widetilde{w} \in \widetilde{W}
\end{array}
$$

where $\widetilde{\boldsymbol{\rho}}$ are auxiliary variables, representing the node potentials.

In the above, constraints (15)-(17) ensure flow balance. Constraint (18) suggests that the link flow of each E-E pair is nonnegative. Constraint (19) implies that the AV link flow is the summation of link flow for different E-E pairs. Constraints (20) and (21) are complementary slackness conditions, specifying that marginal travel times of utilized paths between an E-E pair are the same, but less than or equal to that of any unutilized path.

\subsection{Mixed routing equilibrium}

Definition 1. At the mixed routing equilibrium, for the same mode, perceived travel times of utilized paths between an O-D pair are the same, but less than or equal to that of any unutilized usable path between the same O-D pair.

In the above definition, perceived travel times for CVs are their actual trip times, while the ones for AVs are the actual travel time spent outside of the AV zone, plus the perceived travel times spent within the zone. Recall that the latter is equivalent to the minimum travel time between $A V s$ ' corresponding entrances and exits of the AV zone (see Assumption i).

Mathematically, we can define the mixed routing equilibrium conditions (MRE) for the original network as (1)-(21). Specifically, (6)-(14) specify that, given the perceived travel times within the AV zone, the flow distribution must satisfy the network equilibrium conditions for the revised network; (1)-(5) ensure that AVs' perceived travel times within the AV zone equal the minimum travel times between their corresponding entrances and exits of the zone; (15)-(21) imply that, within the AV zone, AVs must follow the system-optimum routing principle.

To further formulate an equivalent mixed routing equilibrium model, we define a set $\Lambda=$ $\{(\boldsymbol{v}, \boldsymbol{x}, \widetilde{\boldsymbol{\rho}}, \boldsymbol{z}, \boldsymbol{\tau})\}$, where the vector satisfies the following conditions:

$$
\begin{aligned}
& (1),(2),(6)-(10),(18),(19) \\
& \tilde{\rho}_{i}^{\widetilde{w}} \geq 0
\end{aligned} \quad \forall i \in \widetilde{N}, \widetilde{W} \in \widetilde{W}
$$




$$
\tau_{i}^{\widetilde{w}} \geq 0 \quad \forall i \in \widetilde{N} \backslash d(\widetilde{w}), \widetilde{w} \in \widetilde{W}
$$

where $\tau$ are auxiliary variables introduced to facilitate formulating the problem as follows.

Proposition 1. The mixed routing equilibrium conditions (1)-(21) are equivalent to finding $\left(\boldsymbol{v}^{*}, \boldsymbol{x}^{*}, \widetilde{\boldsymbol{\rho}}^{*}, \mathbf{z}^{*}, \boldsymbol{\tau}^{*}\right) \in \Lambda$, which solves the following variational inequality:

MRE-VI:

$\sum_{a \in A} t_{a}\left(v_{a}^{*}\right)\left(v_{a}-v_{a}^{*}\right)+\sum_{a \in \hat{A}}\left[\sum_{\widetilde{w} \in \widetilde{W}} \beta_{a}^{\widetilde{w}} \sum_{\tilde{a} \in \tilde{A}} t_{\tilde{a}}\left(v_{\tilde{a}}^{*}\right) z_{\tilde{a}}^{\widetilde{w}^{*}}\right]\left(v_{a}-v_{a}^{*}\right)+\sum_{\widetilde{w}} \sum_{\tilde{a} \in \tilde{A}}\left[t_{\tilde{a}}\left(v_{\tilde{a}}^{*}\right)+\right.$ $\left.v_{\tilde{a}}^{*} t_{\tilde{a}}^{\prime}\left(v_{\tilde{a}}^{*}\right)-\left(\tilde{\rho}_{i}^{\widetilde{W} *}-\tilde{\rho}_{j}^{\widetilde{W} *}\right)\right]\left(x_{\tilde{a}}^{\widetilde{w}}-x_{\tilde{a}}^{\widetilde{W} *}\right)+\sum_{\widetilde{w}}\left[\left(\sum_{j} x_{o(\widetilde{w}), j}^{\widetilde{w} *}-\sum_{k} x_{k, o(\widetilde{w})}^{\widetilde{w} *}\right)-\right.$ $\left.\sum_{a \in \hat{A}} \beta_{a}^{\widetilde{w}} \sum_{w \in W} x_{a}^{w, A *}\right]\left(\tilde{\rho}_{o(\widetilde{w})}^{\widetilde{w}}-\tilde{\rho}_{o(\widetilde{w})}^{\widetilde{w} *}\right)-\sum_{\widetilde{w}}\left[\left(\sum_{j} x_{o(\widetilde{w}), j}^{\widetilde{w} *}-\sum_{k} x_{k, o(\widetilde{w})}^{\widetilde{w} *}\right)-\right.$ $\left.\sum_{a \in \hat{A}} \beta_{a}^{\widetilde{w}} \sum_{w \in W} x_{a}^{w, A *}\right]\left(\tau_{o(\widetilde{w})}^{\widetilde{w}}-\tau_{o(\widetilde{w})}^{\widetilde{w} *}\right)+\sum_{\widetilde{w}} \sum_{i \in \widetilde{N} \backslash\{o(\widetilde{w}), d(\widetilde{w})\}}\left(\sum_{j} x_{i, j}^{\widetilde{W} *}-\sum_{k} x_{k, i}^{\widetilde{W} *}\right)\left(\tilde{\rho}_{i}^{\widetilde{W}}-\tilde{\rho}_{i}^{\widetilde{W} *}\right)-$ $\sum_{\widetilde{w}} \sum_{i \in \widetilde{N} \backslash\{o(\widetilde{w}), d(\widetilde{w})\}}\left(\sum_{j} x_{i, j}^{\widetilde{W} *}-\sum_{k} x_{k, i}^{\widetilde{W} *}\right)\left(\tau_{i}^{\widetilde{W}}-\tau_{i}^{\widetilde{W} *}\right)+\sum_{\widetilde{w}} \sum_{\tilde{a} \in \tilde{A}} t_{\tilde{a}}\left(v_{\tilde{a}}^{*}\right)\left(z_{\tilde{a}}^{\widetilde{w}}-z_{\tilde{a}}^{\widetilde{W} *}\right) \geq$ $0, \forall(\boldsymbol{v}, \boldsymbol{x}, \widetilde{\boldsymbol{\rho}}, \boldsymbol{z}, \boldsymbol{\tau}) \in \Lambda$

The equivalence can be established by expressing the optimality conditions of MRE-VI and comparing them with the defined mixed routing equilibrium conditions, i.e., MRE. See the appendix for a proof.

Proposition 2. MRE-VI has at least one solution.

Proof: According to the appendix, we know that $\boldsymbol{\tau}$ are nonnegative auxiliary variables that are only used to guarantee the flow balance within the AV network, i.e., (A.44), (A.47), and (A.48); thus, adding some upper bounds to $\boldsymbol{\tau}$ will not affect the other optimal solutions $\left(\boldsymbol{v}^{*}, \boldsymbol{x}^{*}, \widetilde{\boldsymbol{\rho}}^{*}, \mathbf{z}^{*}\right)$. Furthermore, since $\widetilde{\boldsymbol{\rho}}$ represent node potentials, we can always find some upper bounds for them, within which optimal values of these multipliers still exist. As a result, we can construct a restricted MRE-VI by adding corresponding upper bounds to $(\widetilde{\boldsymbol{\rho}}, \boldsymbol{\tau})$. In addition, link flows $\boldsymbol{x}$ and $\boldsymbol{v}$ are bounded, and the upper bound of $\boldsymbol{z}$ is $\mathbf{1}$, which has been demonstrated in Section 3.1. Therefore, the restricted MRE-VI problem has a compact and convex feasible region. Given that all the functions are continuous, the restricted MRE-VI admits at least one solution (see, e.g., Harker and Pang, 1990), so as the original MRE-VI.

However, even if all the link performance functions of both the regular and AV links are strictly monotone, we cannot guarantee the uniqueness of the link flow solution to MRE-VI, as the travel time functions of dummy links (see equality (3)) may not be strictly monotone with respect to the link flows in the revised network.

To illustrate, we consider a simple AV network shown in FIGURE 4(a). The link travel time functions are assumed to be: $t_{12}\left(x_{12}\right)=3 x_{12}, t_{13}\left(x_{13}\right)=3 x_{13}$, and $t_{23}\left(x_{23}\right)=3 x_{23}$, where $x_{12}$, $x_{13}$, and $x_{23}$ are the corresponding link flows. Suppose that 1-2, 1-3, and 2-3 are E-E pairs, then the dummy network is constructed as the same as the original AV network (see FIGURE 4(b)). It is worth pointing out that dummy link 1-3 represents the shorter path of path $1 \rightarrow 3$ and path $1 \rightarrow$ $2 \rightarrow 3$. Let $e_{12}, e_{13}$, and $e_{23}$ denote the dummy flows of link 1-2, 1-3, and 2-3, i.e., the demand of E-E pair 1-2, 1-3, and 2-3, respectively. Furthermore, we assume that $e_{13}>e_{12}+e_{23}$. It is easy to verify that under this assumption, both paths $1 \rightarrow 3$ and $1 \rightarrow 2 \rightarrow 3$ of the AV network will be 
utilized by the trips from E-E pair 1-3. Define the trips using path $1 \rightarrow 2 \rightarrow 3$ as $\hat{e}_{13}$, then the ones using path $1 \rightarrow 3$ are $e_{13}-\hat{e}_{13}$. As a result, we have $x_{12}=e_{12}+\hat{e}_{13}, x_{13}=e_{13}-\hat{e}_{13}$, and $x_{23}=$ $e_{23}+\hat{e}_{13}$. According to the system-optimum routing principle, the marginal cost of path $1 \rightarrow 3$ and $1 \rightarrow 2 \rightarrow 3$ must be equivalent. That is,

$$
6\left(e_{13}-\hat{e}_{13}\right)=6\left(e_{12}+\hat{e}_{13}\right)+6\left(e_{23}+\hat{e}_{13}\right)
$$

which yields $\hat{e}_{13}=\frac{1}{3}\left(e_{13}-e_{12}-e_{23}\right)$.

Based on the flow distribution, we can obtain the travel time functions of the dummy links: $c_{12}=t_{12}\left(x_{12}\right)=2 e_{12}+e_{13}-e_{23}, c_{13}=t_{13}\left(x_{13}\right)=2 e_{13}+e_{12}+e_{23}$, and $c_{23}=t_{23}\left(x_{23}\right)=$ $2 e_{23}+e_{13}-e_{12}$. It is easy to verify that the Jacobian matrix of $\boldsymbol{c}$ with respect to $\boldsymbol{e}$ is only positive semi-definite, rather than positive definite. That is, the travel time functions of dummy links are not strictly monotone with respect to the link flows in the revised network. Consequently, the link flow solution to MRE-VI may not be unique.

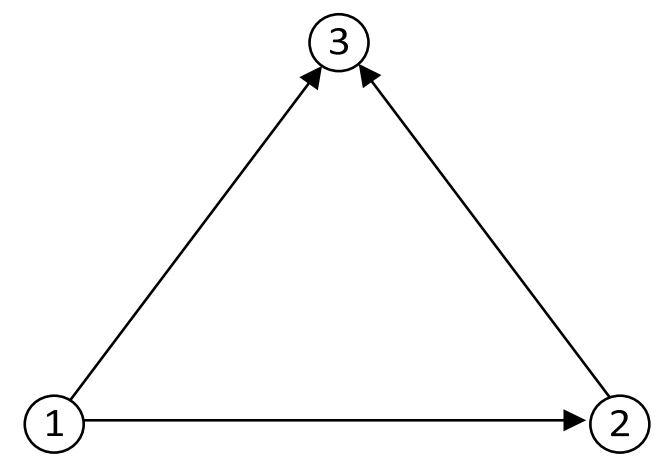

(a) AV network

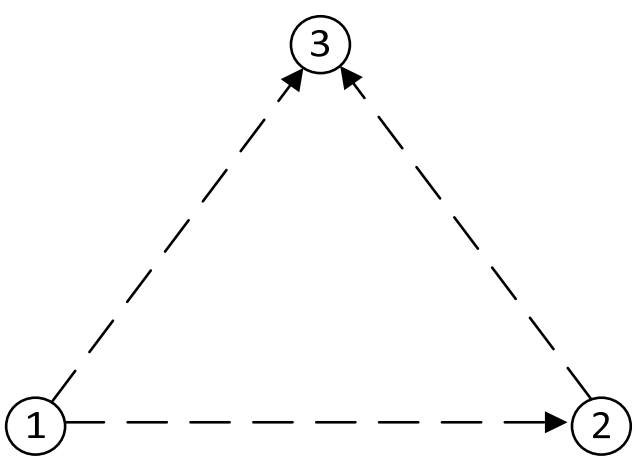

(b) Dummy AV network

FIGURE 4 A simple AV network and its corresponding dummy network

\subsection{Solution procedure}

Although many solution algorithms proposed for solving VI in the literature (see, Facchinei and Pang, 2003) can be applied to solve MRE-VI, they may not be necessarily efficient (Aghassi et al., 2006). In this section, we solve MRE-VI by reformulating it to be the following nonlinear optimization problem via a technique proposed by Aghassi et al. (2006):

MRE-NLP:

$\min _{\boldsymbol{v}, \boldsymbol{x}, \widetilde{\boldsymbol{\rho}}, \mathbf{z}, \widetilde{\boldsymbol{\alpha}}, \widetilde{\boldsymbol{\beta}}, \tilde{\lambda}, \widetilde{\gamma}} \sum_{a \in A} t_{a}\left(v_{a}\right) v_{a}+\sum_{a \in \widehat{A}} c_{a}\left(v_{a}\right) v_{a}+\sum_{\widetilde{w} \in \widetilde{W}} \sum_{\tilde{a} \in \tilde{A}}\left[t_{\tilde{a}}\left(v_{\tilde{a}}\right)+v_{\tilde{a}} t_{\tilde{a}}^{\prime}\left(v_{\tilde{a}}\right)-\left(\tilde{\rho}_{i}^{\widetilde{w}}-\right.\right.$

$\left.\left.\tilde{\rho}_{j}^{\widetilde{w}}\right)\right] x_{\tilde{a}}^{\widetilde{w}}-\sum_{w \in W} \sum_{m \in M} \sum_{i \in N} d_{i}^{w, m} \tilde{\alpha}_{i}^{w, m}-\sum_{\widetilde{w} \in \widetilde{W}}\left(\tilde{\gamma}_{o(\widetilde{w})}^{\widetilde{w}}-\tilde{\gamma}_{d(\widetilde{w})}^{\widetilde{w}}\right)$

s.t. (1), (2), (6)-(10), (18), (19), and (22)
$\tilde{\beta}_{a}=t_{a}\left(v_{a}\right)$
$\tilde{\beta}_{a}=\sum_{\widetilde{w} \in \widetilde{W}} \beta_{a}^{\widetilde{W}} \sum_{\tilde{a} \in \tilde{A}} t_{\tilde{a}}\left(v_{\tilde{a}}\right) z_{\tilde{a}}^{\widetilde{w}}$
$\forall a \in A$
$\tilde{\lambda}_{\tilde{a}}=0$
$\forall a \in \hat{A}$
$\forall \tilde{a} \in \tilde{A}$ 


$$
\begin{array}{ll}
\tilde{\alpha}_{i}^{w, A}-\tilde{\alpha}_{j}^{w, A}-\tilde{\beta}_{a} \leq 0 & \forall a \in A \cup \hat{A}, w \in W \\
\tilde{\alpha}_{i}^{W, C}-\tilde{\alpha}_{j}^{w, C}-\tilde{\beta}_{a} \leq 0 & \forall a \in A, w \in W \\
-\tilde{\lambda}_{\tilde{a}} \leq t_{\tilde{a}}\left(v_{\tilde{a}}\right)+v_{\tilde{a}} t_{\tilde{a}}^{\prime}\left(v_{\tilde{a}}\right)-\tilde{\rho}_{i}^{\widetilde{w}}+\tilde{\rho}_{j}^{\widetilde{w}} & \forall \tilde{a} \in \tilde{A}, \widetilde{w} \in \widetilde{W} \\
\left(\sum_{j} x_{o(\widetilde{w}), j}^{\widetilde{w}}-\sum_{k} x_{k, o(\widetilde{w})}^{\widetilde{w}}\right)-\sum_{a \in \hat{A}} \beta_{a}^{\widetilde{w}} \sum_{w \in W} x_{a}^{w, A}=0 & \forall \widetilde{w} \in \widetilde{W} \\
\sum_{j} x_{i, j}^{\widetilde{w}}-\sum_{k} x_{k, i}^{\widetilde{w}}=0 & \forall i \in \widetilde{N} \backslash\{o(\widetilde{w}), d(\widetilde{w})\}, \widetilde{w} \in \widetilde{W} \\
\tilde{\gamma}_{i}^{\widetilde{w}}-\tilde{\gamma}_{j}^{\widetilde{w}} \leq t_{\tilde{a}}\left(v_{\tilde{a}}\right) & \forall \tilde{a} \in \tilde{A}, \widetilde{w} \in \widetilde{W}
\end{array}
$$

where $\tilde{\alpha}_{i}^{w, A}, \tilde{\alpha}_{i}^{w, C}, \tilde{\beta}_{a}, \tilde{\lambda}_{\tilde{a}}$, and $\tilde{\gamma}_{i}^{\widetilde{w}}$ are auxiliary variables, and $d_{i}^{w, m}=d^{w, m}$, if $i=o(w) ; d_{i}^{w, m}=$ $-d^{w, m}$, if $i=d(w)$; otherwise, $d_{i}^{w, m}=0$.

Specifically, if the optimal value of MRE-NLP is 0 , then its solution $\left(\boldsymbol{v}^{*}, \boldsymbol{x}^{*}, \widetilde{\boldsymbol{\rho}}^{*}, \boldsymbol{z}^{*}, \boldsymbol{\tau}^{*}\right)$ is also the one to MRE-VI.

\subsection{Numerical example}

In this section, numerical examples are conducted based on the network in FIGURE 1. Specifically, there are two O-D pairs with demands shown in TABLE 1, and the link performance function is assumed to be $t_{a}\left(v_{a}\right)=a_{0}+b_{0} \times v_{a}$ min, where $a_{0}$ and $b_{0}$ are provided in TABLE 2 . We construct an AV zone as per FIGURE 1 . That is, nodes 2, 3, 4, 5, and 6 are AV nodes. Accordingly, links 2-3, 2-4, 2-5, 3-4, 3-6, 4-3, 4-5, 4-6, 5-4, and 5-6 are all AV links. It is worthwhile to highlight that since node 4 is neither an origin nor a destination, the dummy network and revised network are constructed as FIGURE 2(a) and FIGURE 3, respectively. As per Assumption iii, we assume that the per-lane capacity becomes triple when a regular link is converted to an AV link. Hence, the AV link performance function is $t_{\tilde{a}}\left(v_{\tilde{a}}\right)=a_{0}+\frac{b_{0}}{3} \times v_{\tilde{a}} \min$.

\section{TABLE 1 O-D demand}

\begin{tabular}{ccc}
\hline O-D & CV & AV \\
\hline $1-7$ & 40 & 30 \\
$8-7$ & 25 & 15 \\
\hline
\end{tabular}

TABLE 2 Network characteristics

\begin{tabular}{ccccccccc}
\hline Link & $a_{0}(\min )$ & $b_{0}$ & Link & $a_{0}(\min )$ & $b_{0}$ & Link & $a_{0}(\min )$ & $b_{0}$ \\
\hline $1-2$ & 1.00 & 1.00 & $3-4$ & 1.50 & 1.00 & $5-4$ & 1.00 & 1.00 \\
$1-3$ & 2.00 & 3.00 & $3-6$ & 1.00 & 1.50 & $5-6$ & 1.00 & 1.00 \\
$1-5$ & 1.00 & 1.00 & $3-7$ & 1.00 & 1.00 & $5-7$ & 2.00 & 2.00 \\
$2-3$ & 2.00 & 1.00 & $4-3$ & 0.50 & 1.00 & $6-7$ & 2.00 & 2.00 \\
$2-4$ & 1.00 & 0.50 & $4-5$ & 1.00 & 1.00 & $8-1$ & 1.00 & 1.00 \\
$2-5$ & 1.00 & 1.00 & $4-6$ & 1.00 & 2.50 & $8-5$ & 2.00 & 4.00 \\
\hline
\end{tabular}


Given the above setting, we obtain the equilibrium solution by solving MRE-NLP. Specifically, TABLE 3 and TABLE 4 display the equilibrium link flows for the original network (see FIGURE 1) and the dummy network (see FIGURE 2(a)), respectively. As we can see, since CVs are not permitted to use the AV links, the equilibrium link flows on the AV links and dummy AV links are all 0. As mentioned previously, the demand for each E-E pair equals the flow of the corresponding dummy AV link. Therefore, the equilibrium link flows in TABLE 4 are also the EE demand for the AV zone. Given the E-E demand, we obtain a system-optimum solution within the AV zone shown in TABLE 5 and TABLE 6. Comparing TABLE 3 with TABLE 5, it is easy to verify that the AV-link flows in TABLE 3 exactly follow the system-optimum flow distribution, which is consistent with the operation concept iii that AVs are routed to minimize the total travel time in the AV zone. Furthermore, making a comparison between TABLE 4 and TABLE 6, we can readily observe that the travel time of each dummy link in TABLE 4 is equal to the minimum path travel time (i.e., the path travel times that are bold and underlined) of the corresponding E-E pair. For example, there are three paths $2 \rightarrow 3,2 \rightarrow 4 \rightarrow 3$, and $2 \rightarrow 5 \rightarrow 4 \rightarrow 3$ between E-E pair 2-3, whose travel times are 7.16, 6.91, and $7.41 \mathrm{~min}$, respectively (see TABLE 6). As expected, the travel time of dummy link 2-3 is $6.91 \mathrm{~min}$ (see TABLE 4), which equals the minimum of the three, i.e., 6.91. Also, from TABLE 6, we can observe the difference between perceived and actual travel times for each E-E pair. For example, for E-E pair 2-3, the actual travel time for path $2 \rightarrow 3$ is equal to $7.16 \mathrm{~min}, 0.25 \mathrm{~min}$ greater than the perceived travel time, i.e., $6.91 \mathrm{~min}$. The maximum discrepancy between perceived and actual travel times appears in E-E pair 2-6, which is 0.5 min, or $5.81 \%$.

TABLE 3 Equilibrium link flow for the original network

\begin{tabular}{cccccccc}
\hline Link & CV flow & AV flow & $\begin{array}{c}\text { Travel time } \\
(\mathrm{min})\end{array}$ & Link & CV flow & AV flow & $\begin{array}{c}\text { Travel time } \\
(\mathrm{min})\end{array}$ \\
\hline $1-2$ & 0.00 & 34.06 & 35.06 & $4-3$ & 0.00 & 9.32 & 3.61 \\
$1-3$ & 23.96 & 0.00 & 73.87 & $4-5$ & 0.00 & 0.00 & 1.00 \\
$1-5$ & 28.81 & 7.84 & 37.65 & $4-6$ & 0.00 & 5.16 & 5.30 \\
$2-3$ & 0.00 & 15.48 & 7.16 & $5-4$ & 0.00 & 0.65 & 1.22 \\
$2-4$ & 0.00 & 13.82 & 3.30 & $5-6$ & 0.00 & 15.05 & 6.02 \\
$2-5$ & 0.00 & 4.76 & 2.59 & $5-7$ & 41.04 & 0.00 & 84.09 \\
$3-4$ & 0.00 & 0.00 & 1.50 & $6-7$ & 0.00 & 22.09 & 46.18 \\
$3-6$ & 0.00 & 1.88 & 1.94 & $8-1$ & 12.76 & 11.91 & 25.67 \\
$3-7$ & 23.96 & 22.91 & 47.87 & $8-5$ & 12.24 & 3.09 & 63.32 \\
\hline
\end{tabular}


TABLE 4 Equilibrium link flow for the dummy network

\begin{tabular}{cccccc}
\hline Link & AV flow & Travel time $(\mathrm{min})$ & Link & AV flow & Travel time (min) \\
\hline $2-3$ & 22.91 & 6.91 & $3-6$ & 0.00 & 1.94 \\
$2-5$ & 0.32 & 2.59 & $5-3$ & 0.00 & 4.82 \\
$2-6$ & 10.83 & 8.60 & $5-6$ & 11.25 & 6.02 \\
$3-5$ & 0.00 & 2.50 & & & \\
\hline
\end{tabular}

TABLE 5 System-optimum link flow pattern within the AV zone

\begin{tabular}{cccccc}
\hline Link & AV flow & Travel time $(\mathrm{min})$ & Link & AV flow & Travel time (min) \\
\hline $2-3$ & 15.48 & 7.16 & $4-3$ & 9.32 & 3.61 \\
$2-4$ & 13.82 & 3.30 & $4-5$ & 0.00 & 1.00 \\
$2-5$ & 4.76 & 2.59 & $4-6$ & 5.16 & 5.30 \\
$3-4$ & 0.00 & 1.50 & $5-4$ & 0.65 & 1.22 \\
$3-6$ & 1.88 & 1.94 & $5-6$ & 15.05 & 6.02 \\
\hline
\end{tabular}

TABLE 6 System-optimum path flow pattern within the AV zone

\begin{tabular}{clccc}
\hline E-E & \multicolumn{1}{c}{ Path } & Path flow & Path travel time (min) & $\begin{array}{c}\text { Marginal path travel } \\
\text { time (min) }\end{array}$ \\
\hline $2-3$ & $2-3$ & 13.60 & 7.16 & 12.32 \\
& $2-4-3$ & 9.32 & $\underline{\mathbf{6 . 9 1}}$ & 12.32 \\
& $2-5-4-3$ & 0.00 & 7.41 & 12.32 \\
\hline $2-5$ & $2-5$ & 0.32 & $\underline{\mathbf{2 . 5 9}}$ & 4.17 \\
& $2-3-4-5$ & 0.00 & 9.66 & 14.82 \\
& $2-4-5$ & 0.00 & 4.30 & 6.61 \\
\hline $2-6$ & $2-3-4-6$ & 0.00 & 13.96 & 23.42 \\
& $2-3-4-5-6$ & 0.00 & 15.67 & 25.85 \\
& $2-3-6$ & 1.88 & 9.10 & 15.20 \\
& $2-4-3-6$ & 0.00 & 8.85 & 15.20 \\
& $2-4-5-6$ & 0.00 & 10.32 & 17.64 \\
& $2-4-6$ & 4.51 & $\underline{\mathbf{8 . 6 0}}$ & 15.20 \\
& $2-5-4-3-6$ & 0.00 & 9.35 & 15.20 \\
& $2-5-4-6$ & 0.65 & 9.10 & 15.20 \\
& $2-5-6$ & 3.79 & $\underline{\mathbf{8 . 6 0}}$ & 15.20 \\
\hline $3-5$ & $3-4-5$ & 0.00 & $\underline{\mathbf{2 . 5 0}}$ & 2.50 \\
\hline $3-6$ & $3-4-5-6$ & 0.00 & 8.52 & 13.53 \\
& $3-4-6$ & 0.00 & $\mathbf{6 . 8 0}$ & 11.10 \\
& $3-6$ & 0.00 & $\underline{\mathbf{1 . 9 4}}$ & 2.88 \\
\hline $5-3$ & $5-4-3$ & 0.00 & $\underline{\mathbf{4 . 8 2}}$ & 8.15 \\
\hline $5-6$ & $5-4-3-6$ & 0.00 & 6.77 & 11.03 \\
\hline
\end{tabular}




\begin{tabular}{lccc}
\hline $5-4-6$ & 0.00 & 6.52 & 11.03 \\
$5-6$ & 11.25 & $\underline{\mathbf{6 . 0 2}}$ & 11.03 \\
\hline
\end{tabular}

TABLE 7 shows the perceived travel times of CVs and AVs with and without the AV zone. As we can see, without the AV zone, both CVs and AVs perceive the same travel time for the same O-D pair, as they share the same road network and link performance functions. However, with the $\mathrm{AV}$ zone deployed, the perceived travel times of AVs between both O-D pairs decrease substantially (e.g., by $19 \%$ between O-D pair 1-7), while the ones of CVs increase considerably (e.g., by $10 \%$ between O-D pair 1-7). This is due to the fact that the AV zone can be utilized only by AVs. Furthermore, as the per-lane capacity of links within the AV zone is assumed to be much larger than those of regular links due to vehicle automation (see Assumption iii), the total travel time with the AV zone is thus expected to be reduced. It can be seen from TABLE 8 that with the presence of the AV zone, the system travel time decreases from 13,202.75 min to 12,987.27 min. Besides the total system travel time, AV-zone planners may also want to analyze the impact of the $\mathrm{AV}$ zone on the AV-zone area (i.e., the area consisting of links 2-3, 2-4, 2-5, 3-4, 3-6, 4-3, 4-5, 46, 5-4, and 5-6). TABLE 8 shows that the total travel time within the AV-zone area decreases substantially, from 1,193.09 min to $324.69 \mathrm{~min}$. The above findings imply that deploying an AV zone may improve the performance of the AV-zone area as well as the whole system.

TABLE 7 Perceived travel times with and without the AV zone

\begin{tabular}{cccc}
\hline Scenario & O-D & $\begin{array}{c}\text { Perceived travel } \\
\text { time of CV (min) }\end{array}$ & $\begin{array}{c}\text { Perceived travel } \\
\text { time of AV (min) }\end{array}$ \\
\hline \multirow{2}{*}{ Without AV zone } & $1-7$ & 110.88 & 110.88 \\
\cline { 2 - 4 } & $1-8$ & 136.04 & 136.04 \\
\hline \multirow{2}{*}{ With AV zone } & $1-7$ & 121.74 & 89.84 \\
\cline { 2 - 4 } & $1-8$ & 147.40 & 115.51 \\
\hline
\end{tabular}

TABLE 8 System and AV-zone area travel times with and without the AV zone

\begin{tabular}{ccc}
\hline Scenario & System travel time (min) & $\begin{array}{c}\text { Travel time within the AV-zone } \\
\text { area (min) }\end{array}$ \\
\hline Without AV zone & $13,202.75$ & $1,193.09$ \\
With AV zone & $12,987.27$ & 324.69 \\
\hline
\end{tabular}

\subsection{Discussions}

The mixed routing equilibrium model discussed above may become more relevant with the deployment of various advanced traffic control and management strategies leveraging connected and automated vehicle technologies. The modeling framework proposed in this paper can be applied to various scenarios where vehicles adopt different routing principles at different sub- 
networks, as long as the routing strategies and the perceived travel times within the sub-networks are well defined. Below is a detailed discussion:

\section{- $\quad$ Routing strategy within the sub-network}

As per the operation concept iii, the control center of the sub-network is assumed to route vehicles to minimize the total travel time in the sub-network. In practice, the control center may have different routing strategies for different sub-networks, such as minimizing vehicle-miles traveled or traffic emissions. Admittedly, there are potential liability and other issues with specifying routes for individual vehicles. Thus the control center could simply apply "selfish routing", i.e., allowing AVs to choose their routes in a user-optimum manner even within the AV sub-network. The proposed model can be readily extended to consider variant routing strategies as long as the following conditions are satisfied:

i. The routing objective function is convex with respect to link flows within the subnetwork.

ii. All constraints are linear.

With the above conditions being met, the routing problem within the sub-network is a convex problem and can be readily embedded into the mixed routing equilibrium model. More specifically, let $\psi$ denote the optimality conditions of the convex problem, and then the mixed routing equilibrium conditions can be represented by $\{(1)-(14), \boldsymbol{y} \in \psi\}$, where $\boldsymbol{y}$ is the optimization variable vector. Consequently, the proposed model can be applied to multiple sub-networks with different routing strategies directly.

\section{- Perceived travel time within the sub-network}

According to Assumption i, the perceived travel times are assumed to be the minimum travel times between the corresponding entrances and exits of the sub-network, which implies an optimistic attitude of vehicles in perceiving their travel time when entering the AV zone. Other considerations can be accommodated. For example, the longest travel times (without loop) between the entrances and exits can be taken as the perceived travel times within the sub-network, which implies a pessimistic routing behavior. However, the consideration needs to ensure the perceived travel times to be uniquely defined. For example, the average travel time between an entrance and an exit appears a good proxy for the perceived travel time. Unfortunately, the value depends on the path flow distribution and may not be uniquely determined under the system-optimum routing principle. Consequently, taking it as the perceived travel time might lead to one driver having different perceived travel times even if the link flow distribution is given. In this situation, there may be an infinite number of network equilibrium flow patterns, which would impose a challenge for various

planning applications that rely on the typically unique equilibrium flow distribution as the sole estimate or forecast of how traffic will react to changes in the transportation system. Additional 
care needs to be exercised to handle such situations (see, e.g., Lou et al., 2010; Ban et al., 2013; de Andrade et al., 2016).

\section{OPTIMAL DESIGN OF AUTONOMOUS VEHICLE ZONE}

Given the proposed mixed routing equilibrium model, we proceed to optimize the deployment plan of an AV zone over a general network. As previously stated, the problem can be formulated as a mixed-integer bi-level programming model. The lower-level problem is the mixed routing equilibrium model developed above, i.e., MRE-VI. In the upper-level problem, the decision variables specify where to set up the AV zone, i.e., which links are upgraded to be AV links. All AV links should be clustered and cordoned off by a virtual loop. When a link becomes an AV link, its per-lane capacity will be increased to a given value. The objective is to minimize the social cost, which consists of the construction cost, the total system travel time, and the loss of social welfare due to the loss of accessibility for some CV drivers. Mathematically, it is to minimize $\sum_{\tilde{a} \in \tilde{A}} S_{\tilde{a}}+$ $\sum_{a \in A} t_{a}\left(v_{a}\right) v_{a}+\sum_{\tilde{a} \in \tilde{A}} t_{\tilde{a}}\left(v_{\tilde{a}}\right) v_{\tilde{a}}+\sum_{w \in W} \varrho^{w} \zeta^{w} d^{w, C}$, where $s_{\tilde{a}}$ is the construction cost for AV link $\tilde{a} ; \varrho^{w}=1$, if the accessibility for O-D pair $w$ is damaged by the presence of the AV zone, and 0 otherwise; $\zeta^{w}$ is the loss of benefit for a CV driver between O-D pair $w$ due to the loss of accessibility.

\subsection{Solution procedure}

Although the problem is NP-hard, a few heuristic algorithms can be applied to solve it effectively, such as those in Zhang and Yang (2004), Sumalee (2004), and Hult (2006). However, most of these existing heuristic algorithms may not generate new feasible design plans efficiently, with a few exceptions (e.g., Sumalee, 2004), where complicated strategies have been developed to ensure the feasibility of new design plans.

The simulated annealing algorithm or SAA is a probabilistic method proposed by Kirkpatrick et al. (1983) and Cerny (1985) for finding the global optimum of a given function. Its basic idea is to consider a neighboring solution of the current solution at each step, and apply a probability function to decide whether to move to the new solution or not. It stops until a maximum number of iterations is reached. This study applies SAA to solve the optimal design model, as an efficient procedure of finding new feasible design plans can be encapsulated in it. Specifically, the AV zone starts from a random single node within the candidate area, and then, as per SAA, is expanded by converting a neighboring non-AV node (i.e., the preceding or succeeding non-AV nodes of AV nodes, see FIGURE 5) within the candidate area to an AV node (we refer to the new AV zone as the "neighboring AV zone") at each iteration. To verify whether the neighboring AV zone is feasible, i.e., being surrounded by a closed cordon, the cutset-based algorithm proposed by Zhang and Yang (2004) can be applied. Specifically, a cutset of a graph is defined as "a minimal collection of links whose removal reduces the rank of the graph by one (and only one)." If the cut that separates the AV zone and non-AV zone is a cutset, then a new feasible design plan is generated; otherwise, another neighboring AV zone will be considered. Doing so leads to a better efficiency of finding new feasible plans, as the probability of a neighboring AV zone being a closed cordon is very high. 


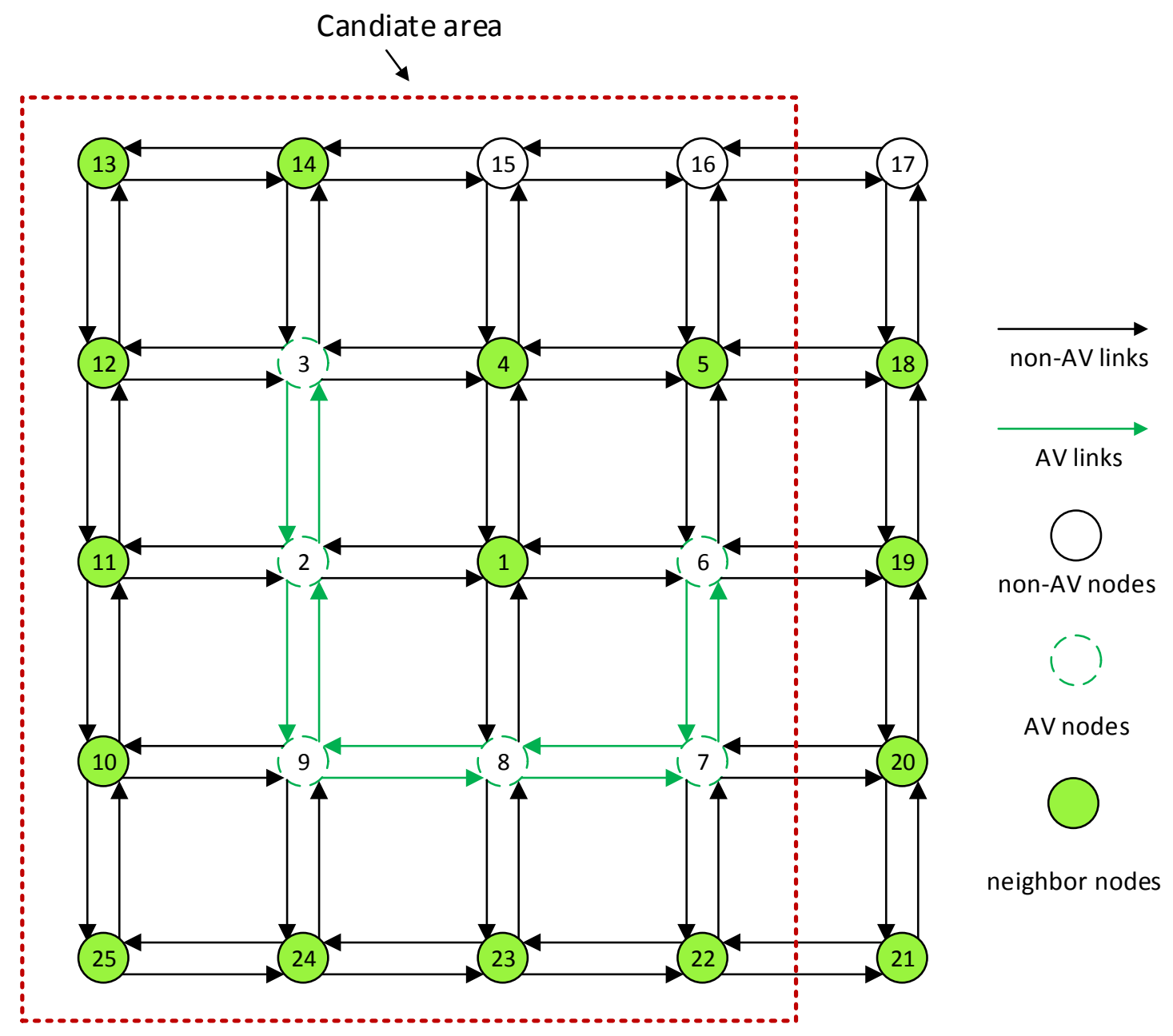

FIGURE 5 A sample AV zone

\subsection{Numerical example}

In this section, numerical examples are conducted based on a network with 81 nodes and 288 links (see FIGURE 6). The dotted red line illustrates the candidate area where AV nodes can only be located. The Bureau of Public Roads (BPR) function, $t_{a}=t_{a}^{0}\left(1+0.15\left(\frac{v_{a}}{c_{a}}\right)^{4}\right)$ is adopted as the link performance function, where $t_{a}^{0}$ and $C_{a}$ are free-flow travel time and capacity of link $a$, respectively. Their values are randomly generated from intervals $(5,20)$ and $(5,100)$, respectively. In particular, as per Assumption iii, we assume that the per-lane capacity triples when a regular link is converted to an AV link. The O-D demand is displayed in TABLE 9. Without losing generality, we assume that the construction cost for $\mathrm{AV}$ zones is 0 .

The optimal AV-zone design is shown in FIGURE 6. Observed from the AV zone, interestingly, it is unlike the best tolling cordons found by Zhang and Yang (2004) and Sumalee (2004), which are rounded; instead, it has a relatively long and narrow shape. Such a design can prevent $\mathrm{CVs}$ from detouring too much while providing privilege for $\mathrm{AVs}$, as CVs can drive across the $\mathrm{AV}$ zone via particular $\mathrm{AV}$ nodes (e.g., node 2 and 13; recall that $\mathrm{CV}$ s are forbidden to use $\mathrm{AV}$ 
links instead of AV nodes). For example, path $55 \rightarrow 30 \rightarrow 13 \rightarrow 14 \rightarrow 3 \rightarrow 2 \rightarrow 9 \rightarrow 24 \rightarrow 23 \rightarrow$ $22 \rightarrow 21 \rightarrow 42 \rightarrow 71$ is an available path for CVs from origin 55 to destination 71 . Therefore, the $\mathrm{AV}$ zone design appears reasonable, as, while reducing the travel cost of $\mathrm{AVs}$, it will not compromise the welfare of CVs too much, and may even improve it.

TABLE 10 shows the travel costs with and without the AV zone deployed. Specifically, with the AV zone deployed, the system travel cost is reduced from 4,169,761 min to 3,278,468 min. That is, the AV zone has reduced the social cost by $21.4 \%$. As mentioned previously, AV-zone planners may be interested in the cost within the AV-zone area. As shown in TABLE 10, the travel cost within the AV-zone area has been reduced by $57.5 \%$. In addition, with the AV zone deployed, the travel cost outside the AV-zone area decreases by $16.8 \%$, although it is not as significant as the one within the AV-zone area. For an additional illustration, FIGURE 7 plots the travel cost saving distribution of CVs. The travel cost savings for all CV trips are positive, which implies that no $\mathrm{CV}$ will suffer from the deployment of the AV zone. That is, such a designed AV zone reduces the travel cost of AVs as well as that of CVs, which is in agreement with the discussion in the previous paragraph. 


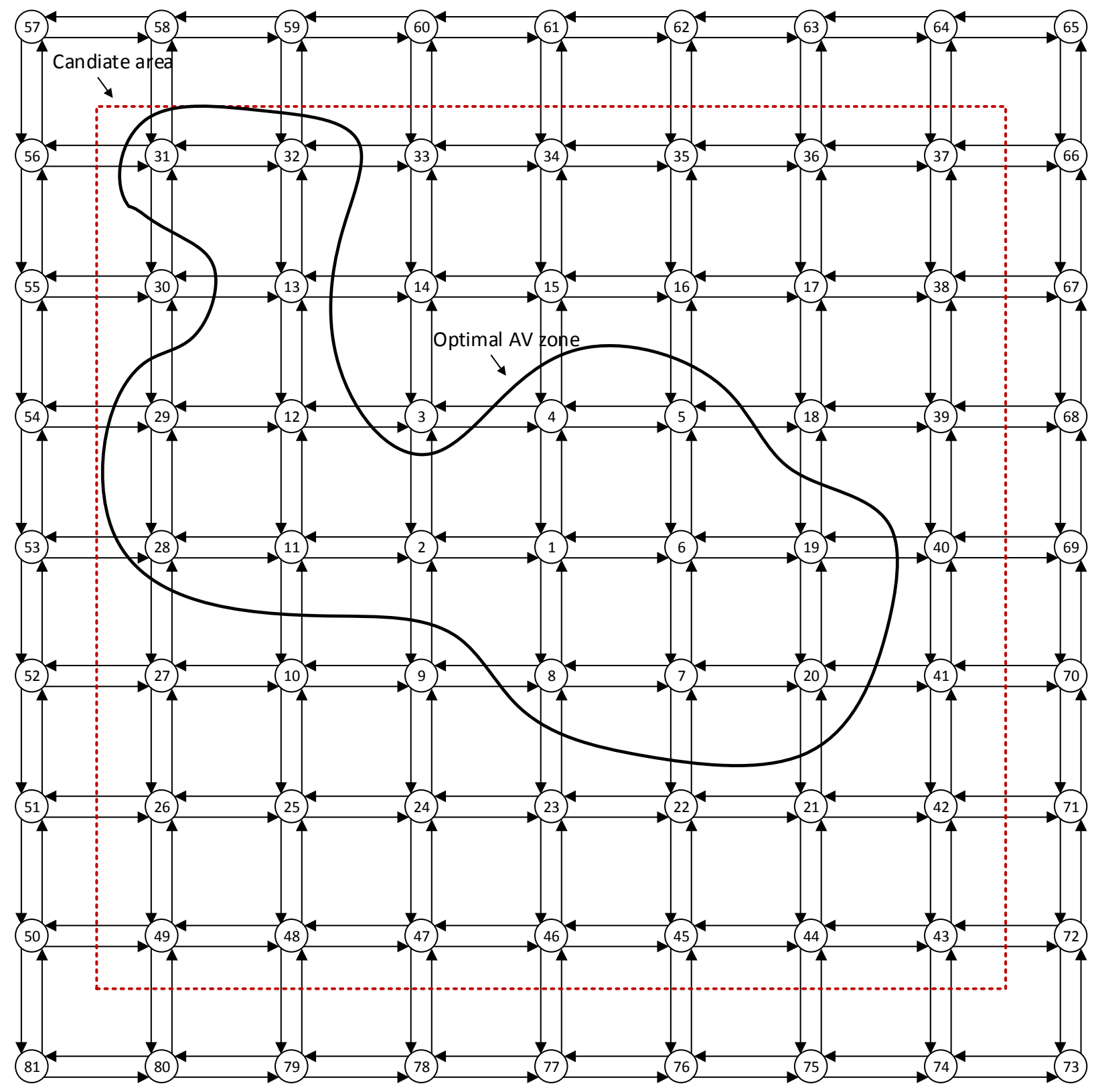

FIGURE 6 Network for the AV zone design 
TABLE 9 O-D demand

\begin{tabular}{ccccccccc}
\hline O-D & CV & AV & O-D & CV & AV & O-D & CV & AV \\
\hline $55-71$ & 20 & 24 & $58-73$ & 20 & 24 & $72-59$ & 30 & 36 \\
$55-72$ & 30 & 36 & $58-74$ & 30 & 36 & $73-55$ & 20 & 24 \\
$55-73$ & 40 & 48 & $58-75$ & 30 & 36 & $73-56$ & 30 & 36 \\
$55-74$ & 20 & 24 & $59-71$ & 20 & 24 & $73-57$ & 40 & 48 \\
$55-75$ & 20 & 24 & $59-72$ & 30 & 36 & $73-58$ & 20 & 24 \\
$56-71$ & 30 & 36 & $59-73$ & 40 & 48 & $73-59$ & 20 & 24 \\
$56-72$ & 20 & 24 & $59-74$ & 20 & 24 & $74-55$ & 30 & 36 \\
$56-73$ & 20 & 24 & $59-75$ & 20 & 24 & $74-56$ & 20 & 24 \\
$56-74$ & 30 & 36 & $71-55$ & 20 & 24 & $74-57$ & 20 & 24 \\
$56-75$ & 30 & 36 & $71-56$ & 30 & 36 & $74-58$ & 30 & 36 \\
$57-71$ & 20 & 24 & $71-57$ & 40 & 48 & $74-59$ & 30 & 36 \\
$57-72$ & 30 & 36 & $71-58$ & 20 & 24 & $75-55$ & 20 & 24 \\
$57-73$ & 40 & 48 & $71-59$ & 20 & 24 & $75-56$ & 30 & 36 \\
$57-74$ & 20 & 24 & $72-55$ & 30 & 36 & $75-57$ & 40 & 48 \\
$57-75$ & 20 & 24 & $72-56$ & 20 & 24 & $75-58$ & 20 & 24 \\
$58-71$ & 30 & 36 & $72-57$ & 20 & 24 & $75-59$ & 20 & 24 \\
$58-72$ & 20 & 24 & $72-58$ & 30 & 36 & & & \\
\hline
\end{tabular}

TABLE 10 Travel costs with and without the AV zone

\begin{tabular}{cccc}
\hline Scenario & $\begin{array}{c}\text { System travel cost } \\
(\mathrm{min})\end{array}$ & $\begin{array}{c}\text { Travel cost within the } \\
\text { AV-zone area (min) }\end{array}$ & $\begin{array}{c}\text { Travel cost outside of } \\
\text { the AV-zone area (min) }\end{array}$ \\
\hline Without AV zone & $4,169,761$ & 471,755 & $3,698,005$ \\
With AV zone & $3,278,468$ & 200,688 & $3,077,780$ \\
\hline
\end{tabular}




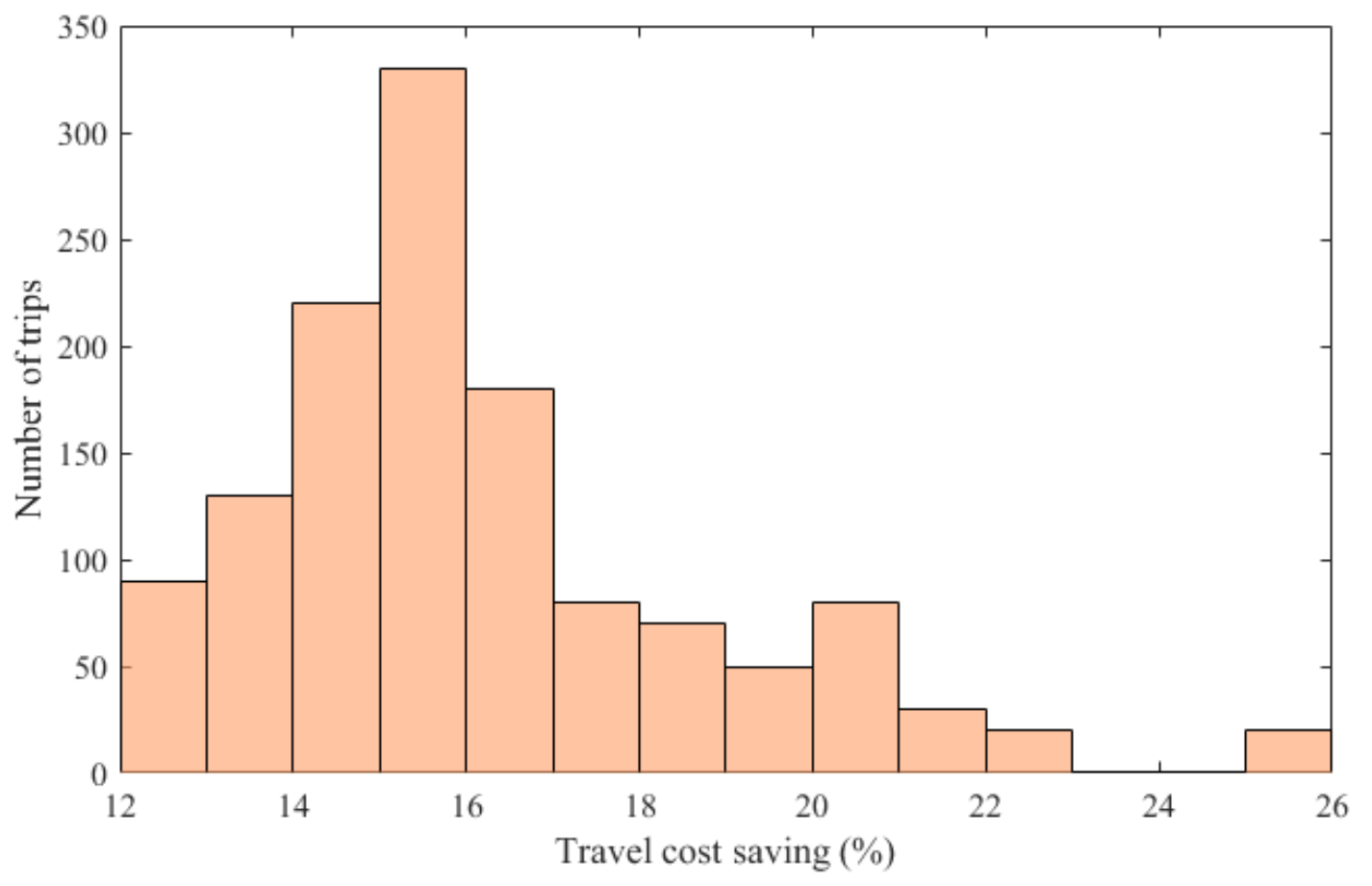

FIGURE 7 Travel cost saving distribution of CVs

\section{CONCLUSION}

Envisioning that $\mathrm{AVs}$ will be deployed in the future and government agencies can dedicate certain areas as AV zones to further promote the adoption of AVs as well as enhance the transportation network performance, this study developed a mathematical framework to optimally design AV zones. To this end, an innovative mixed routing equilibrium model was firstly proposed to describe the flow distribution of $\mathrm{CVs}$ and $\mathrm{AVs}$ with the presence of $\mathrm{AV}$ zones on a road network. Specifically, different from the traditional mixed equilibrium model where each type of player only obeys a particular routing principle across the whole network, AVs apply the user-optimum routing principle when outside of the AV zones, but follow the system-optimum routing principle within the AV zones. This results in a mixed routing behavior for AVs. To capture such a phenomenon, a dummy network was constructed to replace the original AV network where each dummy link represents a set of paths connecting an entrance and an exit of the AV zone; accordingly, the travel cost of each dummy link is in fact the travel cost of the associated entrance and exit pair. As a result, formulating the mixed routing equilibrium model across the original network is equivalent to establishing a traditional network equilibrium model on the revised network. With the established mixed routing equilibrium model, a mixed-integer bi-level programming model was proposed to obtain the optimal design plan of AV zones. The SAA heuristic algorithm was then adopted to solve the model efficiently. Numerical examples show that the social cost may be reduced substantially by an optimal deployment of AV zones. 


\section{AKNOWLEDGEMENTS}

The work described in this paper was partly supported by research grants from the National Science Foundation (CMMI-1362631; CMMI-1562420) and the Southeastern Transportation Research, Innovation, Development \& Education Center (STRIDE).

\section{REFERENCE}

Aghassi, M., Bertsimas, D., and Perakis, G., 2006. Solving asymmetric variational inequalities via convex optimization. Operations Research Letters, 34, 481-490.

Ban, X. J., Ferris, M., Tang, L., and Lu, S., 2013. Risk-neutral second best toll pricing. Transportation Research Part B, 48, 67-87.

Cerny, V., 1985. A thermodynamic approach to the traveling salesman problem: An efficient simulation. Journal of Optimization Theory and Applications, 45, 41-51.

Chen, Z., He, F., Zhang, L., and Yin, Y., 2016. Optimal deployment of autonomous vehicle lanes with endogenous market penetration. Transportation Research Part C, 72, 143-156.

Correia, G. H. d. A., and van Arem, B., 2016. Solving the User Optimum Privately Owned Automated Vehicles Assignment Problem (UO-POAVAP): A model to explore the impacts of self-driving vehicles on urban mobility. Transportation Research Part B, 87, 64-88.

de Andrade, G. R., Chen, Z., Elefteriadou, L., and Yin, Y., 2016. Multiclass traffic assignment problem with flow-dependent passenger car equivalent (PCE) value of trucks. Transportation Research Record (under review).

Davis, M., 2014. Integrating autonomous vehicles and managed lanes. http://www.rsandh.com/blog/2014-06-19-integrating-autonomous-vehicles-and-managed-lanes2/ (accessed August 18, 2016).

Facchinei, F., and Pang, J.-S., 2003. Finite-dimensional variational inequalities and complementarity problem. Springer, New York.

Godsmark, P., and Kakkar, G., 2014. Why automated vehicle zones make sense. The Canadian Automated Vehicles Centre of Excellence.

https://www.itscanada.ca/files/1_CVAV1_Godsmark Why\%20Automated\%20Vehicle\%20Zone s\%20Make\%20Sense.pdf (accessed August 18, 2016).

Google Self-Driving Car Project, 2016. https://www.google.com/selfdrivingcar/ (accessed October 20, 2016).

Harker, P. T., 1988. Multiple equilibrium behaviors on networks. Transportation Science, 22, 3946.

Harker, P. T., and Pang, J. S., 1990. Finite-dimensional variational inequality and nonlinear complementarity problems: a survey of theory, algorithms and applications. Mathematical Programming, 48, 161-220. 
Haurie, A., and Marcotte, P., 1985. On the relationship between Nash-Cournot and Wardrop equilibria. Networks, 15, 295-308.

He, F., Yin, Y., Shirmohammadi, N., and Y. Nie., 2013. Tradable credit schemes on networks with mixed equilibrium Behaviors. Transportation Research Part B, 57, 47-65.

Hult, E. E., 2006. Closed charging cordon design problem. University of Cambridge, UK, 2006.

Kesting, A., Treiber, M., and Helbing, D., 2010. Enhanced intelligent driver model to access the impact of driving strategies on traffic capacity. Philosophical Transactions of the Royal Society of London A, 368, 4585-4605.

Kirkpatrick, S., Gelatt, C. D., and Vecchi, M. P., 1983. Optimization by simulated annealing. Science, 220, 621-630.

Levin, M. W., and Boyles, S. D., 2015. Effects of autonomous vehicle ownership on trip, mode, and route choice. Transportation Research Record, 2493, 29-38.

Levin, M., and Boyles, S., 2016a. A multiclass cell transmission model for shared human and autonomous vehicle roads. Transportation Research Part C, 62, 103-116.

Levin, M., and Boyles, S., 2016b. A cell transmission model for dynamic lane reversal with autonomous vehicles. Transportation Research Part C, 68, 126-143.

Lou, Y., Yin, Y., and Lawphongpanich, S., 2010. Robust congestion pricing under boundedly rational user equilibrium. Transportation Research Part B, 44, 15-28.

nuTonomy, 2016. http://nutonomy.com/press.html (accessed August 25, 2016).

Shladover, S., Su, D., and Lu, X. Y., 2012. Impacts of cooperative adaptive cruise control on freeway traffic flow. Transportation Research Record, 2324, 63-70.

Sumalee, A., 2004. Optimal road user charging cordon design: A heuristic optimization approach. Computer-Aided Civil and Infrastructure Engineering, 19, 377-392.

Yang, H., and Zhang, X., 2008. Existence of anonymous link tolls for system optimum on networks with mixed equilibrium behaviors. Transportation Research Part B, 42, 99-112.

Zhang, X., and Yang, H., 2004. The optimal cordon-based network congestion pricing problem. Transportation Research Part B, 38, 517-537.

Zhang, X., Yang, H., Huang, H., 2008. Multiclass multicriteria mixed equilbirum on networks and uniform link tolls for system optimum. European Journal of Operational Research, 189, 146-158.

\section{APPENDIX}

This appendix includes the proof of proposition 1. For convenience, we rewrite $\Lambda=$ $\{(\boldsymbol{v}, \boldsymbol{x}, \widetilde{\boldsymbol{\rho}}, \boldsymbol{z}, \boldsymbol{\tau})\}$ as follows:

$$
\boldsymbol{\Delta} \boldsymbol{x}^{w, m}=\boldsymbol{E}^{w, m} \boldsymbol{d}^{w, m} \quad \forall m \in M, w \in W
$$




$$
\begin{array}{ll}
v_{a}=\sum_{w \in W} \sum_{m \in M} x_{a}^{w, m} & \forall a \in A \cup \hat{A} \\
v_{\tilde{a}}=\sum_{\widetilde{w} \in \widetilde{W}} x_{\tilde{a}}^{\widetilde{w}} & \forall \tilde{a} \in \tilde{A} \\
x_{a}^{w, A} \geq 0 & \forall a \in A \cup \hat{A}, w \in W \\
x_{a}^{w, C} \geq 0 & \forall a \in A, w \in W \\
x_{a}^{w, C}=0 & \forall a \in \hat{A}, w \in W \\
x_{\tilde{\tilde{w}}}^{\widetilde{W}} \geq 0 & \forall \tilde{a} \in \tilde{A}, \widetilde{W} \in \widetilde{W} \\
\tilde{\rho}_{i}^{\widetilde{w}} \geq 0 & \forall i \in \widetilde{N}, \widetilde{w} \in \widetilde{W} \\
\tau_{i}^{\widetilde{w}} \geq 0 & \forall i \in \widetilde{N} \backslash d(\widetilde{w}), \widetilde{w} \in \widetilde{W} \\
\tilde{\Delta} \mathbf{z}^{\widetilde{w}}=\tilde{E}^{\widetilde{w}} & \forall \widetilde{w} \in \widetilde{W} \\
z_{\tilde{a}}^{\widetilde{w}} \geq 0 & \forall \tilde{a} \in \tilde{A}, \widetilde{w} \in \widetilde{W}
\end{array}
$$

The optimality conditions of MRE-VI can be stated as follows:

$$
\begin{aligned}
& \text { (A.1)-(A.11) } \\
& \sum_{\widetilde{w} \in \widetilde{W}}^{t_{a}\left(v_{a}\right)-\gamma_{a}=0} \beta_{a}^{\widetilde{w}} \sum_{\tilde{a} \in \tilde{A}} t_{\tilde{a}}\left(v_{\tilde{a}}\right) z_{\tilde{a}}^{\widetilde{w}}-\gamma_{a}=0 \\
& -\gamma_{\tilde{a}}=0 \\
& -\rho_{i}^{w, m}+\rho_{j}^{w, m}+\gamma_{a}-\xi_{a}^{w, m}=0 \\
& t_{\tilde{a}}\left(v_{\tilde{a}}\right)+v_{\tilde{a}} t_{\tilde{a}}^{\prime}\left(v_{\tilde{a}}\right)-\tilde{\rho}_{i}^{\widetilde{w}}+\tilde{\rho}_{j}^{\widetilde{w}}+\gamma_{\tilde{a}}-\mu_{\tilde{a}}^{\widetilde{w}}=0 \\
& -\left(\sum_{j} x_{o(\widetilde{w}), j}^{\widetilde{w}}-\sum_{k} x_{k, o(\widetilde{w})}^{\widetilde{W}}\right)+\sum_{a \in \hat{A}} \beta_{a}^{\widetilde{w}} \sum_{w \in W} x_{a}^{w, A}-\varsigma_{o(\widetilde{w})}^{\widetilde{W}}=0 \\
& \left(\sum_{j} x_{i, j}^{\widetilde{w}}-\sum_{k} x_{k, i}^{\widetilde{w}}\right)-\theta_{i}^{\widetilde{w}}=0 \\
& \left(\sum_{j} x_{i, j}^{\widetilde{w}}-\sum_{k} x_{k, i}^{\widetilde{w}}\right)-\varsigma_{i}^{\widetilde{w}}=0 \\
& t_{\tilde{a}}\left(v_{\tilde{a}}\right)-\kappa_{i}^{\widetilde{w}}+\kappa_{j}^{\widetilde{w}}-\alpha_{\tilde{a}}^{\widetilde{w}}=0 \\
& x_{a}^{w, A} \xi_{a}^{w, A}=0 \\
& x_{a}^{w, C} \xi_{a}^{w, C}=0 \\
& x_{\tilde{a}}^{\widetilde{W}} \mu_{\tilde{a}}^{\widetilde{W}}=0 \\
& \tilde{\rho}_{i}^{\widetilde{w}} \theta_{i}^{\widetilde{w}}=0 \\
& \tau_{i}^{\widetilde{w}} S_{i}^{\widetilde{w}}=0 \\
& z_{\tilde{a}}^{\widetilde{W}} \alpha_{\tilde{a}}^{\widetilde{W}}=0 \\
& \xi_{a}^{w, A} \geq 0 \\
& \xi_{a}^{w, C} \geq 0
\end{aligned}
$$$$
\left(\sum_{j} x_{o(\widetilde{w}), j}^{\widetilde{w}}-\sum_{k} x_{k, o(\widetilde{w})}^{\widetilde{w}}\right)-\sum_{a \in \hat{A}} \beta_{a}^{\widetilde{w}} \sum_{w \in W} x_{a}^{w, A}-\theta_{o(\widetilde{w})}^{\widetilde{w}}=0
$$

$\forall a \in A$

$\forall i$

$\in \widetilde{N} \backslash\{o(\widetilde{w}), d(\widetilde{W})\}$, $\widetilde{W} \in \widetilde{W}$

$\forall i$

$$
\in \widetilde{N} \backslash\{o(\widetilde{W}), d(\widetilde{W})\}
$$$$
\widetilde{w} \in \widetilde{W}
$$

$\forall \tilde{a} \in \tilde{A}, \widetilde{W} \in \widetilde{W}$

$\forall a \in A \cup \hat{A}, w \in W$

$\forall a \in A, w \in W$

$\forall \tilde{a} \in \tilde{A}, \widetilde{w} \in \widetilde{W}$

$\forall i \in \widetilde{N}, \widetilde{w} \in \widetilde{W}$

$\forall i \in \widetilde{N} \backslash d(\widetilde{W})$, $\widetilde{w} \in \widetilde{W}$

$\forall \tilde{a} \in \tilde{A}, \widetilde{w} \in \widetilde{W}$ $\forall a \in A \cup \hat{A}, w \in W$

$\forall a \in A, w \in W$ 
$\mu_{\tilde{a}}^{\widetilde{\widetilde{\omega}}} \geq 0$

$\theta_{i}^{\widetilde{N}} \geq 0$

$S_{i}^{\widetilde{W}} \geq 0$

$\alpha_{\tilde{a}}^{\widetilde{W}} \geq 0$ $\forall \tilde{a} \in \tilde{A}, \widetilde{w} \in \widetilde{W}$

$\forall \widetilde{w} \in \widetilde{W}$

$\forall i \in \widetilde{N} \backslash d(\widetilde{w})$,

$\widetilde{w} \in \widetilde{W}$

$\forall \tilde{a} \in \tilde{A}, \widetilde{w} \in \widetilde{W}$

where $\rho_{i}^{w, m}, \gamma_{a}, \gamma_{\tilde{a}}, \xi_{a}^{w, A}, \mu_{\tilde{a}}^{\widetilde{W}}, \theta_{i}^{\widetilde{w}}, \varsigma_{i}^{\widetilde{w}}, \kappa_{i}^{\widetilde{w}}$, and $\alpha_{\tilde{a}}^{\widetilde{W}}$ are the multipliers of constraints (A.1)-(A.4), and (A.7)-(A.11); $\xi_{a}^{W, C}$ is the multiplier of constraints (A.5) and (A.6).

From (A.12), (A.15), and (A.22), we have:

$\left[t_{a}\left(v_{a}\right)-\rho_{i}^{w, A}+\rho_{j}^{w, A}\right] x_{a}^{w, A}=0, \forall a \in A, w \in W$

From (A.12), (A.15), and (A.28), we have:

$t_{a}\left(v_{a}\right)-\rho_{i}^{w, A}+\rho_{j}^{w, A} \geq 0, \forall a \in A, w \in W$

From (A.12), (A.15), and (A.23), we have:

$\left[t_{a}\left(v_{a}\right)-\rho_{i}^{w, C}+\rho_{j}^{w, C}\right] x_{a}^{w, C}=0, \forall a \in A, w \in W$

From (A.12), (A.15), and (A.29), we have:

$t_{a}\left(v_{a}\right)-\rho_{i}^{w, C}+\rho_{j}^{w, C} \geq 0, \forall a \in A, w \in W$

From (A.13), (A.15), and (A.22), we have:

$\left[\sum_{\widetilde{w} \in \widetilde{W}} \beta_{a}^{\widetilde{w}} \sum_{a \in A} t_{\tilde{a}}\left(v_{\tilde{a}}\right) z_{\tilde{a}}^{\widetilde{W}}-\rho_{i}^{w, A}+\rho_{j}^{w, A}\right] x_{a}^{w, A}=0, \forall a \in \hat{A}, w \in W$

From (A.13), (A.15), and (A.28), we have:

$\sum_{\widetilde{w} \in \widetilde{W}} \beta_{a}^{\widetilde{w}} \sum_{a \in A} t_{\tilde{a}}\left(v_{\tilde{a}}\right) z_{\tilde{a}}^{\widetilde{w}}-\rho_{i}^{w, A}+\rho_{j}^{w, A} \geq 0, \forall a \in \hat{A}, w \in W$

From (A.14), (A.16), and (A.24), we have:

$\left[t_{\tilde{a}}\left(v_{\tilde{a}}\right)+v_{\tilde{a}} t_{\tilde{a}}^{\prime}\left(v_{\tilde{a}}\right)-\tilde{\rho}_{i}^{\widetilde{W}}+\tilde{\rho}_{j}^{\widetilde{w}}\right] x_{\tilde{a}}^{\widetilde{W}}=0, \forall \tilde{a}=(i, j) \in \tilde{A}, \widetilde{w} \in \widetilde{W}$

From (A.14), (A.16), and (A.30), we have:

$t_{\tilde{a}}\left(v_{\tilde{a}}\right)+v_{\tilde{a}} t_{\tilde{a}}^{\prime}\left(v_{\tilde{a}}\right)-\tilde{\rho}_{i}^{\widetilde{w}}+\tilde{\rho}_{j}^{\widetilde{w}} \geq 0, \forall \tilde{a}=(i, j) \in \tilde{A}, \widetilde{w} \in \widetilde{W}$

From (A.17) and (A.31), we have:

$\left(\sum_{j} x_{o(\widetilde{w}), j}^{\widetilde{W}}-\sum_{k} x_{k, o(\widetilde{w})}^{\widetilde{w}}\right)-\sum_{a \in \hat{A}} \beta_{a}^{\widetilde{w}} \sum_{w \in W} x_{a}^{w, A}=\theta_{o(\widetilde{w})}^{\widetilde{w}} \geq 0, \forall \widetilde{w} \in \widetilde{W}$

From (A.18) and (A.32), we have:

$-\left(\sum_{j} x_{o(\widetilde{w}), j}^{\widetilde{w}}-\sum_{k} x_{k, o(\widetilde{w})}^{\widetilde{w}}\right)+\sum_{a \in \hat{A}} \beta_{a}^{\widetilde{w}} \sum_{w \in W} x_{a}^{w, A}=\varsigma_{o(\widetilde{w})}^{\widetilde{w}} \geq 0, \forall \widetilde{w} \in \widetilde{W}$

We know that (A.42) and (A.43) can hold only if $\theta_{o(\widetilde{w})}^{\widetilde{w}}=0, \varsigma_{o(\widetilde{w})}^{\widetilde{w}}=0, \forall \widetilde{W} \in \widetilde{W}$ and 
$\left(\sum_{j} x_{o(\widetilde{w}), j}^{\widetilde{W}}-\sum_{k} x_{k, o(\widetilde{w})}^{\widetilde{W}}\right)=\sum_{a \in \hat{A}} \beta_{a}^{\widetilde{w}} \sum_{w \in W} x_{a}^{w, A}=0, \forall \widetilde{W} \in \widetilde{W}$

From (A.19) and (A.31), we have:

$\left(\sum_{j} x_{i, j}^{\widetilde{w}}-\sum_{k} x_{k, i}^{\widetilde{w}}\right)=\theta_{i}^{\widetilde{w}} \geq 0, \forall i \in \widetilde{N} \backslash\{o(\widetilde{w}), d(\widetilde{w})\}, \widetilde{w} \in \widetilde{W}$

From (A.20) and (A.32), we have:

$\left(\sum_{j} x_{i, j}^{\widetilde{\widetilde{w}}}-\sum_{k} x_{k, i}^{\widetilde{\widetilde{w}}}\right)=\theta_{i}^{\widetilde{\widetilde{w}}} \geq 0, \forall i \in \widetilde{N} \backslash\{o(\widetilde{w}), d(\widetilde{w})\}, \widetilde{w} \in \widetilde{W}$

Similarly, (A.45) and (A.46) can hold only if: $\theta_{i}^{\widetilde{w}}=0, \varsigma_{i}^{\widetilde{w}}=0, \forall \in \widetilde{N} \backslash\{o(\widetilde{w}), d(\widetilde{w})\}, \widetilde{W} \in \widetilde{W}$ and

$\sum_{j} x_{i, j}^{\widetilde{w}}-\sum_{k} x_{k, i}^{\widetilde{w}}=0, \forall i \in \widetilde{N} \backslash\{o(\widetilde{w}), d(\widetilde{w})\}, \widetilde{W} \in \widetilde{W}$

Further, summating (A.44) and (A.47), we can obtain:

$\sum_{j} x_{d(\widetilde{w}), j}^{\widetilde{W}}-\sum_{k} x_{k, d(\widetilde{w})}^{\widetilde{w}}=-\sum_{a \in \hat{A}} \beta_{a}^{\widetilde{w}} \sum_{w \in W} x_{a}^{w, A}$

From (A.21) and (A.27), we have:

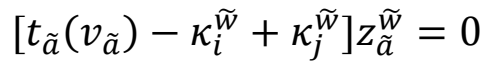

From (A.21) and (A.33), we have:

$t_{\tilde{a}}\left(v_{\tilde{a}}\right)-\kappa_{i}^{\widetilde{w}}+\kappa_{j}^{\widetilde{w}} \geq 0$

Obviously, (A.1)-(A.11), (A.34)-(A.41), (A.44), and (A.47)-(A.50) are equivalent to the MRE conditions (1)-(21).

Note that the optimality condition of MRE-VI contains additional constraints, such as, (A.8), (A.9), (A.25), and (A.26), but this will not affect the equivalence. 